\title{
Article \\ Circle in Space-Space in Circle: A Study of Ratio between Open Space and Built-Up Area in Historical Circular Objects
}

\author{
Biljana Stanislav Jović ${ }^{1, *}$, Aleksandar Acim Čučaković ${ }^{2}$ and Mihailo Nebojsa Grbić ${ }^{1}$ \\ 1 Department of Landscape Architecture and Horticulture, Faculty of Forestry, University of Belgrade, \\ 11000 Belgrade, Serbia; mihailo.grbic@sfb.bg.ac.rs \\ 2 Department of Mathematics, Physics and Descriptive Geometry, Faculty of Civil Engineering, University of \\ Belgrade, 11000 Belgrade, Serbia; cucak@grf.bg.ac.rs \\ * Correspondence: biljana.jovic@sfb.bg.ac.rs; Tel.: +381-60-3541-394
}

Citation: Jović, B.S.; Čučaković, A.A.; Grbić, M.N. Circle in Space-Space in Circle: A Study of Ratio between Open Space and Built-Up Area in Historical Circular Objects. Sustainability 2021, 13, 4662. https:// doi.org/10.3390/su13094662

Academic Editor:

Carmela Cucuzzella

Received: 9 March 2021

Accepted: 16 April 2021

Published: 22 April 2021

Publisher's Note: MDPI stays neutral with regard to jurisdictional claims in published maps and institutional affiliations.

Copyright: (c) 2021 by the authors. Licensee MDPI, Basel, Switzerland. This article is an open access article distributed under the terms and conditions of the Creative Commons Attribution (CC BY) license (https:// creativecommons.org/licenses/by/ $4.0 /)$.

\begin{abstract}
Many cities nowadays explore different circular designs as new sustainable city concepts in different urban aspects. The new trend, as part of the adaptation for climate change, is a strategy of increasing the number of urban open spaces, and circular plan design could be a sustainable approach to urban development. This paper provides a historical overview of various examples of circular objects containing built structures and open spaces from the Neolithic to the present. The Built-Up Area (BUA) and Open Space (OS) relationships are shown histogramically for 36 objects arranged chronologically. The morphospace analysis was performed to determine any possible regularity in the relationships of parts of circular objects. For the purpose of this research, three variables were chosen. First, all selected historical examples of circular objects were divided into two main categories: objects with a total diameter smaller than $300 \mathrm{~m}$ and objects with a total diameter bigger than $300 \mathrm{~m}$. Additionally, the selected circular objects were divided by their type of open space to better understand their spatial position. The largest number of analyzed objects belongs to the Parks-Gardens category, followed by settlements, and then earth works, sacral objects and circular buildings, with the smallest number of circular objects being in the category of allotments and plazas. The second variable was Jam area and \% of Jam. The buildings are of different sizes up to several hundred $\mathrm{m}^{2}$, and the areas range up to several hundred ha. The total area to OS ratio ranges from $0 \%$ (for Large Serdab) to $100 \%$ (for multiple objects). There is a similar situation with the diameter ratios (total and "jam"). Additionally, the final variable was the historical position of the selected circular objects. Circular objects belong to all historical periods from the Neolithic to the present. The aim of this research was to explore the relationship between OS and BUA in various circular objects with different diameters of open spaces and find out if there was any regularity in this relationship. The morphospace analysis of this research indicates that there is no clear regularity in the relationship between the built-up area and the open space, but the aspects and research results shown here contribute to sustainability since the circular design approach could play a key role in future circular design processes.
\end{abstract}

Keywords: circular objects; open space; built-up area; morphospace analysis; doughnut objects

\section{Introduction}

Many cities around the world explore different sustainable strategies (implementing circular economy, circular product design, circular urban systems, ideas of circular cities as a destination of the future) to improve city environment, and circular designs could be a sustainable approach for improving urban ecosystems and a solution for climate change adaptations.

The ratio between porous and non-porous surface areas in cities represents just one of the problems in urban environments. For sustainable urban development, it is important to find a proper ratio between these two urban structures. Different types of open spaces 
have important roles in the urban environment (social, economic and also environmental role)-reducing negative effects of urban development, such as surface runoff and the urban heat island effect, and many others [1]. There are many definitions of open spaces. One definition defines open spaces as area with its surface open to the sky, regulated to serve conservation and urban shaping function in addition to providing recreational opportunities and many others [2]. In many definitions, open spaces are in unbreakable connection with green areas and natural spaces, so they often represent a porous area in a city (but not conditionally - there are many other types of open spaces too).

The concept of circular city and the circular approach in urban planning started at the end of the 20th century, as a contrast to linear planning practice [3]. Today, the circular concept includes more aspects, not only urban planning. The main focus is on circular city design, circular processes and circular management as a way to improve urban functions and protect natural resources. All these goals take place in some urban space. The circularity approach in urban landscape design could have an impact on various sustainable strategies, such as improving waste management and improving traffic paths and communities [4], and have an important role in the adaptation of cities for climate change.

The base of any circular process, circular city or circular object is a circle. Together with the quadrilateral and the cross, the circle belongs to the most basic and oldest symbols. The source of the circle is the point and together they are symbols of perfection: the point because of the nucleus and the source of everything created from it, the circle because it has neither beginning nor end, and speaks of infinite circular motion. In Euclidean geometry, a circle is the geometric location of points in a plane that are at an equal distance from one constant point in the same plane. If perfect is simple, then the circle is indeed the most perfect of all lines, precisely because it is the simplest. The circle is also the basic line in geometry because all other curved lines can be obtained starting from the circle. The circle has been and remains a symbol of perfection in all civilizations [5-8].

From the earliest epochs, the circle was an easily discernible sign that the visual world is obvious. First, the circular forms of the celestial vault, the sun and the full moon, were the most evident sources for their transfer and application into various beliefs. The ancient peoples knew of four key events in the annual cycle of the sun: the winter solstice, the summer solstice, and the spring and autumn equinox. The belief in the protective power of the circle in defense against evil forces, the magic circle, was widespread in many civilizations.

A circle is the only basic geometric shape that a curved line makes. This feature allows the circle to be an element of all artistic styles and trends in architecture and landscape architecture. It fits equally well with the geometric, formal style created primarily from straight lines and polygonal shapes, but also into landscapes or natural gardens.

\section{Materials and Methods}

The objectives of this study are circular objects containing a Built-Up Area (BUA) and an Open Space (OS) from the Neolithic to the present and the exploration of circular doughnut design. In this paper, a Built-Up Area (BUA) represents any land on which buildings and/or other built structures are present-man-made (non-porous) surfaces, and an Open Space (OS) is any piece of land that has no buildings or other built structures, usually a space that is partly or completely covered with grass, trees, shrubs, or other vegetation, with a porous surface-a green space. Circular objects were analyzed using the morphospace method to determine the relationship between an OS and a BUA and ascertain possible regularities in these relationships.

\subsection{The Categories of the Selected Circular Objects and Geometrical Data in Morphospace Analysis}

The morphospace method represents mathematical/graphical representations and is used for studying the evolution of morphological diversity and for the evaluation of evolved shapes among theoretically possible ones. It is a method in theoretical biology for the definition of natural forms of animals and plants $[9,10]$, and in earlier research with the 
application of this method in architecture [11], the relationship between the OS and the BUA is analyzed within circular based objects or, according to Steadman's terminology, the "jam" and the "doughnut".

For morphospace analysis, three variables were chosen. Each axis of the morphospace corresponds to a variable which describes some character of a selected circular object [12]. In this morphospace analysis, there were three selected axes/variables: the first variable is measurements of the area of selected circular spaces (also diameter and inner D), Jam area and $\%$ of the Jam, and the second variable is represented through two main categories with types of circular objects. The third variable represents a historical overview of selected circular objects. All selected objects with their variables are represented in Table 1.

Table 1. The basic data of morphospace analysis of selected circular objects.

\begin{tabular}{|c|c|c|c|c|c|c|c|c|c|}
\hline & Site Name & Dating & Type & Coordinates & Diameter & Inner D & Area & Jam Area & $\%$ of Jam \\
\hline 1. & GöbekliTepe & $10,000 \mathrm{BC}$ & $\begin{array}{l}\text { Sacral } \\
\text { Objects }\end{array}$ & $\begin{array}{l}37^{\circ} 13^{\prime} 23^{\prime \prime} \mathrm{N} \\
38^{\circ} 55^{\prime} 21^{\prime \prime} \mathrm{E}\end{array}$ & $20 \mathrm{~m}$ & $14 \mathrm{~m}$ & $312 \mathrm{~m}^{2}$ & $146 \mathrm{~m}^{2}$ & 46.79 \\
\hline 2. & Stonehenge & $\begin{array}{c}3000-2000 \\
\text { BC }\end{array}$ & Earthworks & $\begin{array}{l}51^{\circ} 10^{\prime} 44^{\prime \prime} \mathrm{N} \\
01^{\circ} 49^{\prime} 34^{\prime \prime} \mathrm{W}\end{array}$ & $110 \mathrm{~m}$ & $109 \mathrm{~m}$ & $9500 \mathrm{~m}^{2}$ & $9354 \mathrm{~m}^{2}$ & 98.46 \\
\hline 3. & Mari & $\begin{array}{c}2900-1759 \\
\text { BC }\end{array}$ & Settlements & $\begin{array}{l}34^{\circ} 55^{\prime} 04^{\prime \prime} \mathrm{N} \\
40^{\circ} 88^{\prime} 91^{\prime \prime} \mathrm{E}\end{array}$ & $2000 \mathrm{~m}$ & $1416 \mathrm{~m}$ & $3,140,000 \mathrm{~m}^{2}$ & $1,574,289 \mathrm{~m}^{2}$ & 50.14 \\
\hline 4. & Arkaim & $\begin{array}{l}2000-1600 \\
\text { BC }\end{array}$ & Settlements & $\begin{array}{l}52^{\circ} 38^{\prime} 57^{\prime \prime} \mathrm{N} \\
59^{\circ} 34^{\prime} 17^{\prime \prime} \mathrm{E}\end{array}$ & $150 \mathrm{~m}$ & $63 \mathrm{~m}$ & $16,917 \mathrm{~m}^{2}$ & $3100 \mathrm{~m}^{2}$ & 18.32 \\
\hline 5. & Mycenean Grave Circle B & $1700 \mathrm{BC}$ & $\begin{array}{l}\text { Sacral } \\
\text { Objects }\end{array}$ & $\begin{array}{l}37^{\circ} 43^{\prime} 51^{\prime \prime} \mathrm{N} \\
22^{\circ} 45^{\prime} 23^{\prime \prime} \mathrm{E}\end{array}$ & $33 \mathrm{~m}$ & $28 \mathrm{~m}$ & $855 \mathrm{~m}^{2}$ & $627 \mathrm{~m}^{2}$ & 73.33 \\
\hline 6. & $\begin{array}{c}\text { Varro's Aviary at Casinum } \\
\text { (Tholos) }\end{array}$ & $64 \mathrm{BC}$ & $\begin{array}{l}\text { Parks- } \\
\text { Gardens }\end{array}$ & $\begin{array}{l}41^{\circ} 29^{\prime} 24^{\prime \prime} \mathrm{N} \\
13^{\circ} 48^{\prime} 50^{\prime \prime} \mathrm{E}\end{array}$ & $14 \mathrm{~m}$ & $9 \mathrm{~m}$ & $223 \mathrm{~m}^{2}$ & $69 \mathrm{~m}^{2}$ & 30.94 \\
\hline 7. & $\begin{array}{c}\text { Mausoleum of Augustus in } \\
\text { Rome }\end{array}$ & $31-28$ BC & $\begin{array}{l}\text { Sacral } \\
\text { Objects }\end{array}$ & $\begin{array}{l}41^{\circ} 54^{\prime} 22^{\prime \prime} \mathrm{N} \\
12^{\circ} 28^{\prime} 35^{\prime \prime} \mathrm{E}\end{array}$ & $84.5 \mathrm{~m}$ & $71 \mathrm{~m}$ & $5600 \mathrm{~m}^{2}$ & $4010 \mathrm{~m}^{2}$ & 71.61 \\
\hline 8. & $\begin{array}{c}\text { Teatro marittimo-Villa } \\
\text { Hadriana }\end{array}$ & $118-138$ & $\begin{array}{l}\text { Circular } \\
\text { Buildings }\end{array}$ & $\begin{array}{l}41^{\circ} 94^{\prime} 24^{\prime \prime} \mathrm{N} \\
12^{\circ} 77^{\prime} 58^{\prime \prime} \mathrm{E}\end{array}$ & $45 \mathrm{~m}$ & $35 \mathrm{~m}$ & $1590 \mathrm{~m}^{2}$ & $972 \mathrm{~m}^{2}$ & 61.131 \\
\hline 9. & Round city of Baghdad & $766-7$ & Settlements & $\begin{array}{l}33^{\circ} 34^{\prime} 88^{\prime \prime} \mathrm{N} \\
44^{\circ} 33^{\prime} 53^{\prime \prime} \mathrm{E}\end{array}$ & $2400 \mathrm{~m}$ & 1717 m & $3,492,672 \mathrm{~m}^{2}$ & $2,316,375 \mathrm{~m}^{2}$ & 66.32 \\
\hline 10. & $\begin{array}{l}\text { Large serdab Dar al-Khalifa, } \\
\text { Samarra }\end{array}$ & 836 & Plazas & $\begin{array}{l}34^{\circ} 23^{\prime} 05^{\prime \prime} \mathrm{N} \\
43^{\circ} 87^{\prime} 40^{\prime \prime} \mathrm{E}\end{array}$ & $50 \mathrm{~m}$ & $0 \mathrm{~m}$ & $1962 \mathrm{~m}^{2}$ & $0 \mathrm{~m}^{2}$ & 0 \\
\hline 11. & Tell al-Banat & $859-892$ & Earthworks & $\begin{array}{l}34^{\circ} 44^{\prime} 76^{\prime \prime} \mathrm{N} \\
43^{\circ} 80^{\prime} 48^{\prime \prime} \mathrm{E}\end{array}$ & $170 \mathrm{~m}$ & $170 \mathrm{~m}$ & $23,584 \mathrm{~m}^{2}$ & $23,584 \mathrm{~m}^{2}$ & 100 \\
\hline 12. & $\begin{array}{l}\text { The Early Slavic pagan } \\
\text { shrine in Peryn }\end{array}$ & $980-989$ & $\begin{array}{l}\text { Sacral } \\
\text { Objects }\end{array}$ & $\begin{array}{l}58^{\circ} 47^{\prime} 28^{\prime \prime} \mathrm{N} \\
31^{\circ} 27^{\prime} 36^{\prime \prime} \mathrm{E}\end{array}$ & $35 \mathrm{~m}$ & $35 \mathrm{~m}$ & $962 \mathrm{~m}^{2}$ & $962 \mathrm{~m}^{2}$ & 100 \\
\hline 13. & Studenica Monastery, Serbia & $1183-1186$ & $\begin{array}{l}\text { Sacral } \\
\text { Objects }\end{array}$ & $\begin{array}{l}43^{\circ} 29^{\prime} 11^{\prime \prime} \mathrm{N} \\
20^{\circ} 32^{\prime} 13^{\prime \prime} \mathrm{E}\end{array}$ & $128 \mathrm{~m}$ & $93 \mathrm{~m}$ & $10,920 \mathrm{~m}^{2}$ & $6851 \mathrm{~m}^{2}$ & 62.74 \\
\hline 14. & Harman Fortified Church & 1240 & $\begin{array}{l}\text { Sacral } \\
\text { Objects }\end{array}$ & $\begin{array}{l}45^{\circ} 42^{\prime} 56^{\prime \prime} \mathrm{N} \\
25^{\circ} 41^{\prime} 06^{\prime \prime} \mathrm{E}\end{array}$ & $100 \mathrm{~m}$ & $63 \mathrm{~m}$ & $6677 \mathrm{~m}^{2}$ & $3115 \mathrm{~m}^{2}$ & 46.65 \\
\hline 15. & $\begin{array}{l}\text { Hypnerotomachia Poliphili } \\
\text { Island garden of Cythera }\end{array}$ & 1499 & $\begin{array}{c}\text { Parks- } \\
\text { Gardens }\end{array}$ & imagination & $1482 \mathrm{~m}$ & $1482 \mathrm{~m}$ & $1,724,114 \mathrm{~m}^{2}$ & $1,724,114 \mathrm{~m}^{2}$ & 100 \\
\hline 16. & Orto Botanico di Padova & 1545 & $\begin{array}{l}\text { Parks- } \\
\text { Gardens }\end{array}$ & $\begin{array}{l}45^{\circ} 39^{\prime} 12^{\prime \prime} \mathrm{N} \\
11^{\circ} 88^{\prime} 08^{\prime \prime} \mathrm{E}\end{array}$ & $84 \mathrm{~m}$ & $64 \mathrm{~m}$ & $5242 \mathrm{~m}^{2}$ & $3205 \mathrm{~m}^{2}$ & 61.14 \\
\hline 17. & Palmanova & 1593 & Settlements & $\begin{array}{l}45^{\circ} 90^{\prime} 56^{\prime \prime} \mathrm{N} \\
13^{\circ} 31^{\prime} 00^{\prime \prime} \mathrm{E}\end{array}$ & $1530 \mathrm{~m}$ & $1115 \mathrm{~m}$ & $1,835,170 \mathrm{~m}^{2}$ & $976,962 \mathrm{~m}^{2}$ & 53.24 \\
\hline 18. & Parc d'Enghien & $1630-1665$ & $\begin{array}{c}\text { Parks- } \\
\text { Gardens }\end{array}$ & $\begin{array}{l}50^{\circ} 68^{\prime} 76^{\prime \prime} \mathrm{N} \\
04^{\circ} 04^{\prime} 73^{\prime \prime} \mathrm{E}\end{array}$ & $235 \mathrm{~m}$ & $163 \mathrm{~m}$ & $41,823 \mathrm{~m}^{2}$ & $20,919 \mathrm{~m}^{2}$ & 50.042 \\
\hline 19. & The Palace Garden Karlsruhe & $1731-1746$ & $\begin{array}{c}\text { Parks- } \\
\text { Gardens }\end{array}$ & $\begin{array}{l}49^{\circ} 01^{\prime} 38^{\prime \prime} \mathrm{N} \\
08^{\circ} 40^{\prime} 44^{\prime \prime} \mathrm{E}\end{array}$ & $865 \mathrm{~m}$ & $589 \mathrm{~m}$ & $575,069 \mathrm{~m}^{2}$ & $272,250 \mathrm{~m}^{2}$ & 47.34 \\
\hline 20. & Clemenswerth Palace, Sögel & $1737-1747$ & $\begin{array}{l}\text { Parks- } \\
\text { Gardens }\end{array}$ & $\begin{array}{l}52^{\circ} 83^{\prime} 83^{\prime \prime} \mathrm{N} \\
07^{\circ} 53^{\prime} 71^{\prime \prime} \mathrm{E}\end{array}$ & $100 \mathrm{~m}$ & $84 \mathrm{~m}$ & $7850 \mathrm{~m}^{2}$ & $5550 \mathrm{~m}^{2}$ & 70.71 \\
\hline 21. & $\begin{array}{c}\text { Schwetzingen Palace, } \\
\text { Schwetzingen }\end{array}$ & $1753-1770$ & $\begin{array}{c}\text { Parks- } \\
\text { Gardens }\end{array}$ & $\begin{array}{l}49^{\circ} 38^{\prime} 37^{\prime \prime} \mathrm{N} \\
08^{\circ} 56^{\prime} 80^{\prime \prime} \mathrm{E}\end{array}$ & $330 \mathrm{~m}$ & $206 \mathrm{~m}$ & $85,476 \mathrm{~m}^{2}$ & $33,299 \mathrm{~m}^{2}$ & 38.96 \\
\hline 22. & $\begin{array}{c}\text { ErylouHua'an tuloi, Hua'an, } \\
\text { Zhangzhou, Fujian, China }\end{array}$ & 1770 & Settlements & $\begin{array}{r}25^{\circ} 01^{\prime} 25^{\prime \prime} \mathrm{N} \\
117^{\circ} 41^{\prime} 12^{\prime \prime} \mathrm{E} \\
\end{array}$ & $73 \mathrm{~m}$ & $27 \mathrm{~m}$ & $4231 \mathrm{~m}^{2}$ & $572 \mathrm{~m}^{2}$ & 13.52 \\
\hline 23. & Șarlota (Charlottenburg) & 1771 & Settlements & $\begin{array}{l}45^{\circ} 97^{\prime} 86^{\prime \prime} \mathrm{N} \\
21^{\circ} 51^{\prime} 91^{\prime \prime} \mathrm{E}\end{array}$ & $417 \mathrm{~m}$ & $326 \mathrm{~m}$ & $136,639 \mathrm{~m}^{2}$ & $83,321 \mathrm{~m}^{2}$ & 60.98 \\
\hline 24. & Circleville, Ohio & 1836 & Earthworks & $\begin{array}{l}39^{\circ} 35^{\prime} 58^{\prime \prime} \mathrm{N} \\
82^{\circ} 56^{\prime} 40^{\prime \prime} \mathrm{W}\end{array}$ & $327 \mathrm{~m}$ & $184 \mathrm{~m}$ & $81,513 \mathrm{~m}^{2}$ & $26,770 \mathrm{~m}^{2}$ & 32.84 \\
\hline
\end{tabular}


Table 1. Cont.

\begin{tabular}{|c|c|c|c|c|c|c|c|c|c|}
\hline & Site Name & Dating & Type & Coordinates & Diameter & Inner D & Area & Jam Area & $\begin{array}{l}\% \text { of } \\
\text { Jam }\end{array}$ \\
\hline 25. & $\begin{array}{l}\text { Queen Mary's Gardens } \\
\text { Regent's Park, London }\end{array}$ & $1930 \mathrm{~s}$ & Parks-Gardens & $\begin{array}{l}51^{\circ} 52^{\prime} 77^{\prime \prime} \mathrm{N} \\
00^{\circ} 15^{\prime} 36^{\prime \prime} \mathrm{W}\end{array}$ & $333 \mathrm{~m}$ & $287 \mathrm{~m}$ & $87,516 \mathrm{~m}^{2}$ & $64,868 \mathrm{~m}^{2}$ & 74.12 \\
\hline 26. & $\begin{array}{l}\text { Brøndby Garden City, } \\
\text { Copenhagen }\end{array}$ & 1964 & Allotments & $\begin{array}{l}55^{\circ} 38^{\prime} 13^{\prime \prime} \mathrm{N} \\
12^{\circ} 23^{\prime} 58^{\prime \prime} \mathrm{E}\end{array}$ & $120 \mathrm{~m}$ & $99 \mathrm{~m}$ & $11,200 \mathrm{~m}^{2}$ & $7720 \mathrm{~m}^{2}$ & 68.93 \\
\hline 27. & Kikar Hamedina, Tel Aviv & 1970 & Plazas & $\begin{array}{l}32^{\circ} 05^{\prime} 12^{\prime \prime} \mathrm{N} \\
34^{\circ} 47^{\prime} 23^{\prime \prime} \mathrm{E}\end{array}$ & $330 \mathrm{~m}$ & $255 \mathrm{~m}$ & $86,484 \mathrm{~m}^{2}$ & $51,250 \mathrm{~m}^{2}$ & 59.26 \\
\hline 28. & $\begin{array}{l}\text { Bublik Circular House, } \\
\text { Moscow }\end{array}$ & 1972 & $\begin{array}{l}\text { Circular } \\
\text { Buildings }\end{array}$ & $\begin{array}{l}55^{\circ} 42^{\prime} 26^{\prime \prime} \mathrm{N} \\
37^{\circ} 28^{\prime} 16^{\prime \prime} \mathrm{E}\end{array}$ & $180 \mathrm{~m}$ & $155 \mathrm{~m}$ & $25,447 \mathrm{~m}^{2}$ & $18,869 \mathrm{~m}^{2}$ & 74.15 \\
\hline 29. & $\begin{array}{c}\text { Maheshwari Udyan, } \\
\text { Mumbai }\end{array}$ & contemporary & Parks-Gardens & $\begin{array}{l}19^{\circ} 02^{\prime} 67^{\prime \prime} \mathrm{N} \\
72^{\circ} 85^{\prime} 54^{\prime \prime} \mathrm{E}\end{array}$ & $150 \mathrm{~m}$ & $104 \mathrm{~m}$ & $20,100 \mathrm{~m}^{2}$ & $8414 \mathrm{~m}^{2}$ & 41.86 \\
\hline 30. & $\begin{array}{c}\text { Gartenverein zur } \\
\text { Sonnenblume Kamp-Lintfort }\end{array}$ & contemporary & Allotments & $\begin{array}{l}51^{\circ} 49^{\prime} 15^{\prime \prime} \mathrm{N} \\
06^{\circ} 56^{\prime} 54^{\prime \prime} \mathrm{E}\end{array}$ & $190 \mathrm{~m}$ & $144 \mathrm{~m}$ & $28,398 \mathrm{~m}^{2}$ & $16,376 \mathrm{~m}^{2}$ & 57.67 \\
\hline 31. & Gasholder Park, London & 2013 & Parks-Gardens & $\begin{array}{l}51^{\circ} 32^{\prime} 13^{\prime \prime} \mathrm{N} \\
0^{\circ} 07^{\prime} 41^{\prime \prime} \mathrm{W}\end{array}$ & $45 \mathrm{~m}$ & $34 \mathrm{~m}$ & $1590 \mathrm{~m}^{2}$ & $927 \mathrm{~m}^{2}$ & 58.30 \\
\hline 32. & $\begin{array}{l}\text { Navy Yard Central Green, } \\
\text { Philadelphia }\end{array}$ & 2015 & Parks-Gardens & $\begin{array}{l}39^{\circ} 89^{\prime} 38^{\prime \prime} \mathrm{N} \\
75^{\circ} 17^{\prime} 12^{\prime \prime} \mathrm{W}\end{array}$ & $113 \mathrm{~m}$ & $81 \mathrm{~m}$ & $10,020 \mathrm{~m}^{2}$ & $5192 \mathrm{~m}^{2}$ & 51.82 \\
\hline 33. & Apple Campus 2, Cupertino & 2017 & $\begin{array}{l}\text { Circular } \\
\text { Buildings }\end{array}$ & $\begin{array}{c}37^{\circ} 33^{\prime} 50^{\prime \prime} \mathrm{N} \\
122^{\circ} 00^{\prime} 90^{\prime \prime} \\
\mathrm{W}\end{array}$ & $461 \mathrm{~m}$ & $348 \mathrm{~m}$ & $167,000 \mathrm{~m}^{2}$ & $95,000 \mathrm{~m}^{2}$ & 56.89 \\
\hline 34. & $\begin{array}{l}\text { Bird enclosure earthwork at } \\
\text { Kickapoo river, Wisconsin }\end{array}$ & $\begin{array}{c}\text { Pre- } \\
\text { Columbian } \\
\text { era }\end{array}$ & Earthworks & $\begin{array}{l}43^{\circ} 28^{\prime} 32^{\prime \prime} \mathrm{N} \\
90^{\circ} 41^{\prime} 38^{\prime \prime} \mathrm{W}\end{array}$ & $118 \mathrm{~m}$ & $118 \mathrm{~m}$ & $10,936 \mathrm{~m}^{2}$ & $10,936 \mathrm{~m}^{2}$ & 100 \\
\hline 35. & $\begin{array}{l}\text { Portsmouth Earthworks } \\
\text { Group C, Kentucky }\end{array}$ & $\begin{array}{c}\text { Pre- } \\
\text { Columbian } \\
\text { era }\end{array}$ & Earthworks & $\begin{array}{l}38^{\circ} 44^{\prime} 09^{\prime \prime} \mathrm{N} \\
82^{\circ} 54^{\prime} 12^{\prime \prime} \mathrm{W}\end{array}$ & $424 \mathrm{~m}$ & $424 \mathrm{~m}$ & $141,196 \mathrm{~m}^{2}$ & $141,196 \mathrm{~m}^{2}$ & 100 \\
\hline 36. & Upper Midwest Circle City & contemporary & Parks-Gardens & $\begin{array}{l}43^{\circ} 04^{\prime} 00^{\prime \prime} \mathrm{N} \\
89^{\circ} 24^{\prime} 00^{\prime \prime} \mathrm{W}\end{array}$ & $500 \mathrm{~km}$ & & $187,753 \mathrm{~km}^{2}$ & $\mathrm{~km}^{2}$ & \\
\hline
\end{tabular}

The prototype is the space in which the elements are concentrically arranged, with the BUA on the outside and the OS inside, or vice versa, to further include those objects where the space is broken into more or less parts (Figure 1), either concentric, radially or irregularly spaced. In such a case, the inner diameter is obtained by summing the surfaces of the individual OS (jam) and calculating the diameter as if the OS were a single circle. At the same time, the percentage of OS in relation to the total area is given. For each of the 36 objects, the surface is shown histogramically in a logarithmic division table.

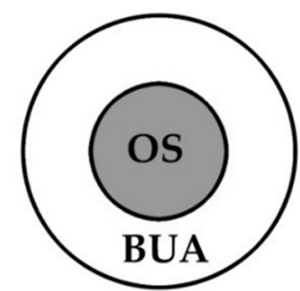

a

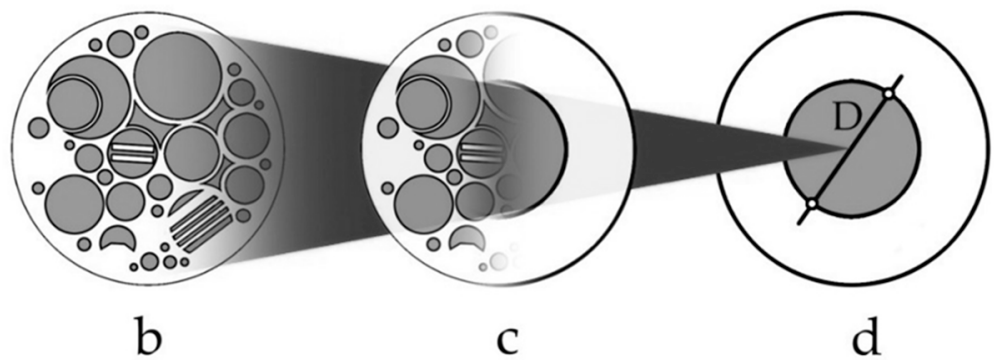

Figure 1. Reduction of complex Open Space (OS) shapes to a single surface to obtain inner diameter for morphospace analysis.

The OS and BUA surfaces are calculated via satellite images from Google Maps using the Photoshop analysis tool. Thirty-six doughnut objects containing a BUA and an OS were analyzed. In addition, other doughnut objects will be mentioned that were not considered because their dimensions could not be obtained, but were significant in the development of some ideas. These are usually descriptions or ideas, often philosophical ones, such as Daedalus' labyrinth, St. Anne's Garden, Civitas veri sive morvm, or the contemporary Circle Cities concept [13]. Within the individual categories, there were a large number of objects, so only the most representative ones were taken (Earthworks, for example). Objects from the Neolithic to the present day, with dimensions ranging from several hundred $\mathrm{m}^{2}$ to 
several hundred ha, have been analyzed. The relative ratios of the bases of the 35 circular objects, as well as the ratios of BUA and OS diameters, or morphospace diagrams, are shown in Figures 2 and 3. One circular object, Upper Midwest Circle City, was excluded as this was a good example of a Circle City idea. Histograms of the BUA and OS surface area relationships arranged chronologically are presented in Figure 4.

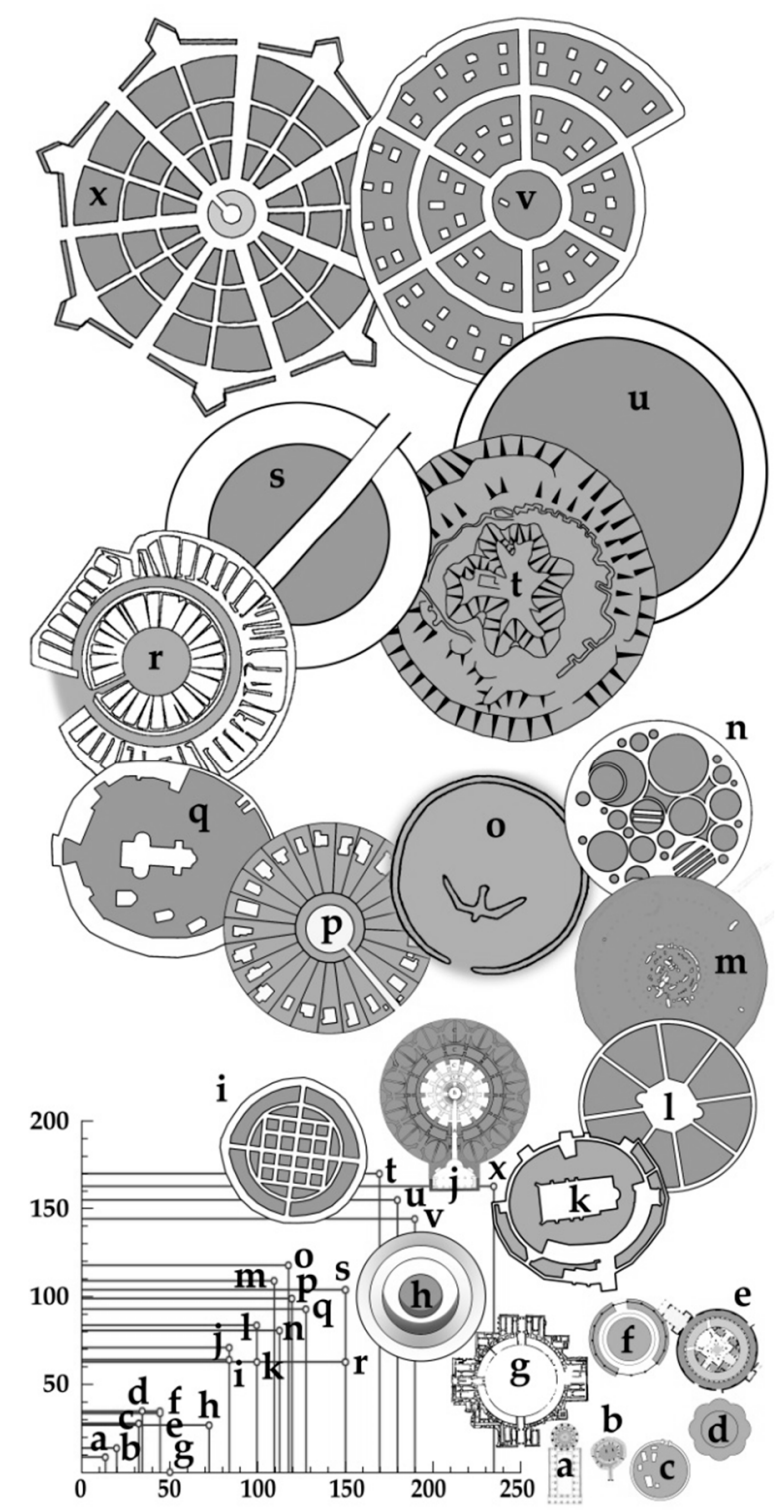

Figure 2. A "morphospace" of diagrammatic plans for circular objects studied (objects with a total diameter smaller than $300 \mathrm{~m}-23$ objects): (a) Varro's Aviary at Casinum, (b) GöbekliTepe, (c) Mycenean Grave Circle B, (d) The Early Slavic pagan shrine in Peryn, (e) Teatro marittimo-Villa Hadriana, (f) Gasholder Park, London, (g) Large serdab Dar al-Khalifa, Samarra, (h) ErylouHua'an tuloi, Hua'an, Zhangzhou, Fujian, China, (i) Orto Botanico di Padova, (j) Mausoleum of Augustus in Rome, (k) Harman Fortified Church, (1) Clemenswerth Palace, Sögel, (m) Stonehenge, (n) Navy Yard Central Green, Philadelphia, (o) Earthwork at Kickapoo river, Wisconsin, (p) Brøndby Garden City, Copenhagen, (q) Studenica Monastery, Serbia, (r) Arkaim, (s) Maheshwari Udyan, Mumbai, (t) Tell al-Banat, (u) Bublik Circular House, Moscow, (v) Gartenverein zur Sonnenblume Kamp-Lintfort, (x) Parc d'Enghien. 


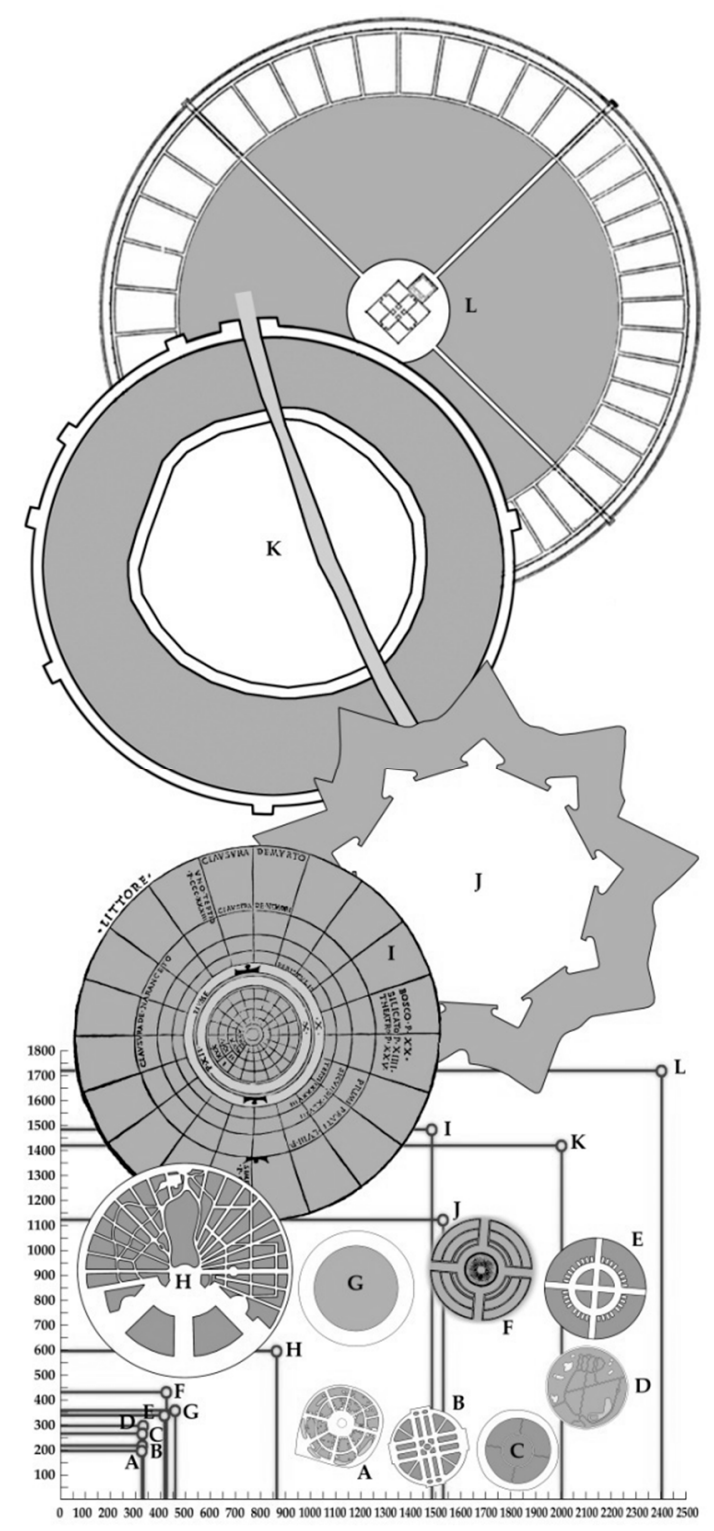

Figure 3. A "morphospace" of diagrammatic plans for circular objects studied (objects with a total diameter bigger than $300 \mathrm{~m}-12$ objects): A-Circleville, Ohio, B-Schwetzingen Palace, Schwetzingen, C-Kikar Hamedina, Tel Aviv, D-Queen Mary's Gardens Regent's Park, London, E-Șarlota (Charlottenburg), F-Portsmouth Earthworks Group C, Kentucky, G-Apple Campus 2, Cupertino, H-The Palace Garden Karlsruhe, I-Island garden of Cythera, J-Palmanova, K-Mari, L-Round city of Baghdad.

In Figure 4, which represents a historical timeline logarithmic histogram of OS and BUAs of selected circular objects analyzing two variables, historical timeline and Jam area, it could be concluded that there are many circular objects where open spaces have larger surface areas than BUAs.

All selected historical examples of circular objects (except Upper Midwest Circle City) were divided into two main categories: objects with a total diameter smaller than $300 \mathrm{~m}$ and objects with a total diameter larger than $300 \mathrm{~m}$. Additionally, the selected circular objects were divided by their type of open space to better understand their spatial position. All the categories are presented in Table 2. 


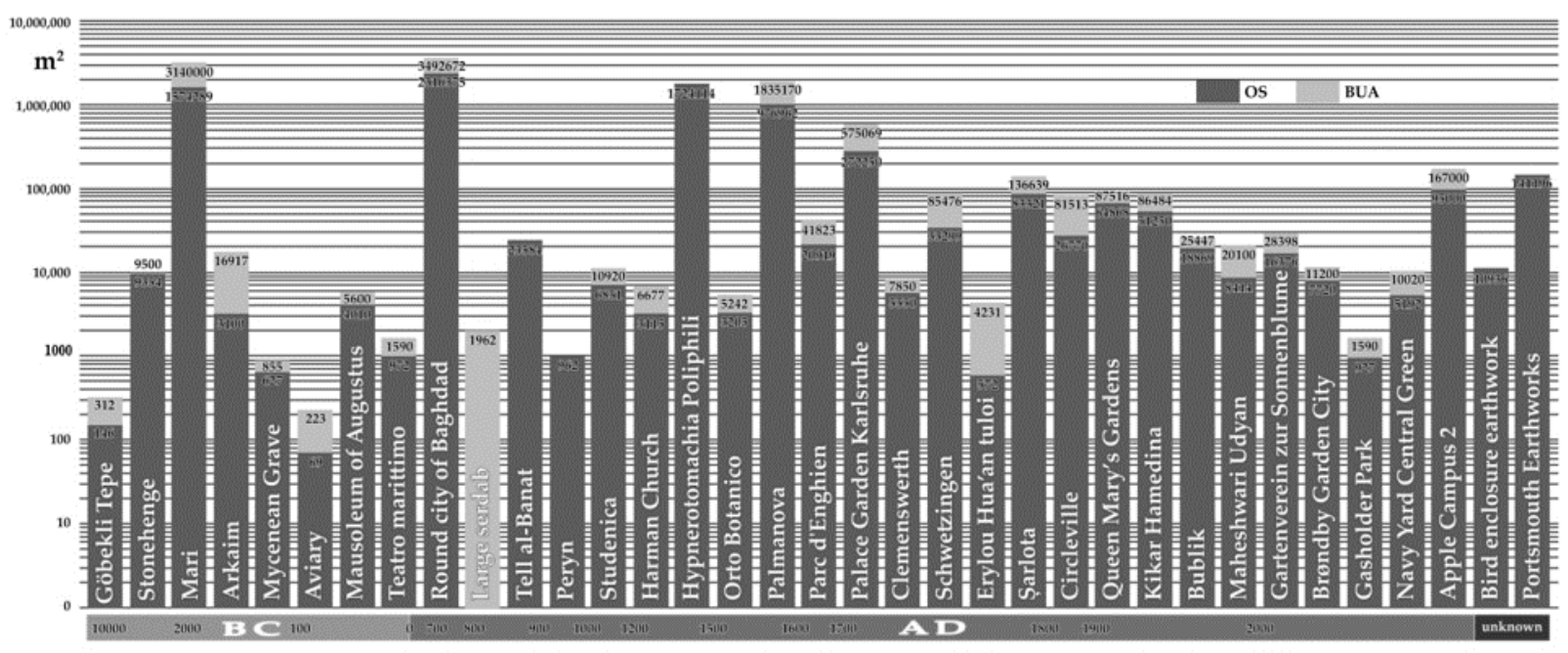

Figure 4. Historical timeline logarithmic histogram of OS and Built-Up Areas (BUAs) of objects analyzed.

Table 2 presents all selected circular objects for this research. For this research, 7 types of circular objects were analyzed: Parks-Gardens (12), Settlements (6), Earthworks (5), Sacral Objects (6), Circular Buildings (3), Allotments (2) and Plazas (2).

Parks-Gardens: Of the 36 circular objects analyzed, 12 belong to the category of green spaces, namely parks or gardens, which occur from ancient times to the present. The oldest object analyzed is Varro's Aviary at Casinum, which was built in 64 BC [14].

During the Renaissance, the idea of circular gardens generally remained unrealized and had a utopian character, such as The Cythera Island garden from the work of Hypnerotomachia Poliphili. One of the few realized gardens is the Orto Botanico di Padova [15].

\subsection{Historical Analysis of the Circular Spaces}

Historical analysis, as a method of the examination of different historical epochs, helps us to better understand the past of these circular objects and the timeline. The timeline is presented in Table 3.

\subsubsection{Neolithic}

According to some opinions, the oldest form of human land-use planning was the garden, a piece of ground fenced off from cattle [16], even preceding the construction of a hut, the beginning of architecture.

In the Americas, there are many traces of circular earthworks, e.g., medicine wheels; Portsmouth Earthworks in Kentucky; the enclosure near the Kickapoo River in Wisconsin [17]; sunken circular plazas from Peru, etc. These creations have been preserved much longer in the New World than in Europe and Asia because of the lifestyle of the indigenous Americans, which was much more tied to nature and did not change much until the arrival of Europeans. With the arrival of European colonizers and the successive urbanization, circular forms were gradually replaced by an orthogonal street layout and building blocks. A good example is the city of Circleville in Ohio. During the 19th century, the Circleville Squaring Company converted a circular earthwork of local native tribes at its center into a squared grid [18].

The Fujian tulou, traditional Fujian earthen buildings, are Chinese rural dwellings unique to south-eastern Fujian, China. One of them has been analyzed, namely the Erylou Hua'an tulou [19]. 
Table 2. The categories of circular objects studied.

\begin{tabular}{|c|c|c|}
\hline Types of Circular Objects & Site Name & Dating \\
\hline & Objects with a Total Diameter Smaller than $300 \mathrm{~m}$ & \\
\hline \multirow{7}{*}{ Parks-Gardens } & Varro's Aviary at Casinum (Tholos) & $64 \mathrm{BC}$ \\
\hline & Maheshwari Udyan, Mumbai & contemporary \\
\hline & Orto Botanico di Padova & 1545 \\
\hline & Parc d'Enghien & $1630-1665$ \\
\hline & Clemenswerth Palace, Sögel & $1737-1747$ \\
\hline & Gasholder Park, London & 2013 \\
\hline & Navy Yard Central Green, Philadelphia & 2015 \\
\hline \multirow{2}{*}{ Settlements } & Arkaim & $2000-1600$ BC \\
\hline & ErylouHua'an tuloi, Hua'an, Zhangzhou, Fujian, China & 1770 \\
\hline \multirow{2}{*}{ Earthworks } & Stonehenge & 3000-2000 BC \\
\hline & Bird enclosure earthwork at Kickapoo river, Wisconsin & Pre-Columbian era \\
\hline & Tell al-Banat & $859-892$ \\
\hline \multirow{6}{*}{ Sacral Objects } & GöbekliTepe & $10,000 \mathrm{BC}$ \\
\hline & Mycenean Grave Circle B & $1700 \mathrm{BC}$ \\
\hline & Mausoleum of Augustus in Rome & $31-28 \mathrm{BC}$ \\
\hline & The Early Slavic pagan shrine in Peryn & 980-989 \\
\hline & Studenica Monastery, Serbia & $1183-1186$ \\
\hline & Harman Fortified Church & 1240 \\
\hline \multirow{2}{*}{ Circular Buildings } & Teatro marittimo-Villa Hadriana & 118-138 \\
\hline & Bublik Circular House, Moscow & 1972 \\
\hline \multirow{2}{*}{ Allotments } & Brøndby Garden City, Copenhagen & 1964 \\
\hline & Gartenverein zur Sonnenblume Kamp-Lintfort & contemporary \\
\hline Plazas & Large serdab Dar al-Khalifa, Samarra & 836 \\
\hline \multicolumn{3}{|c|}{ Objects with a Total Diameter Larger than $300 \mathrm{~m}$} \\
\hline \multirow{4}{*}{ Parks-Gardens } & Schwetzingen Palace, Schwetzingen & 1753-1770 \\
\hline & Queen Mary's Gardens Regent's Park, London & $1930 \mathrm{~s}$ \\
\hline & The Palace Garden Karlsruhe & $1731-1746$ \\
\hline & Hypnerotomachia Poliphili, Island garden of Cythera & 1499 \\
\hline \multirow{4}{*}{ Settlements } & Șarlota (Charlottenburg) & 1771 \\
\hline & Palmanova & 1593 \\
\hline & Mari & 2900-1759 BC \\
\hline & Round city of Baghdad & $766-7$ \\
\hline \multirow{2}{*}{ Earthworks } & Circleville, Ohio & 1836 \\
\hline & Portsmouth Earthworks Group C, Kentucky & Pre-Columbian era \\
\hline Sacral Objects & - & - \\
\hline Circular Buildings & Apple Campus 2, Cupertino & 2017 \\
\hline Allotments & - & - \\
\hline Plazas & Kikar Hamedina, Tel Aviv & 1970 \\
\hline
\end{tabular}


Table 3. The timeline of selected circular objects studied.

\begin{tabular}{|c|c|c|c|}
\hline & Dating & Site Name & Coordinates \\
\hline 1. & $10,000 \mathrm{BC}$ & GöbekliTepe & $37^{\circ} 13^{\prime} 23^{\prime \prime} \mathrm{N} 38^{\circ} 55^{\prime} 21^{\prime \prime} \mathrm{E}$ \\
\hline 2. & 3000-2000 BC & Stonehenge & $51^{\circ} 10^{\prime} 44^{\prime \prime} \mathrm{N} 01^{\circ} 49^{\prime} 34^{\prime \prime} \mathrm{W}$ \\
\hline 3. & $2900-1759$ BC & Mari & $34^{\circ} 55^{\prime} 04^{\prime \prime} \mathrm{N} 40^{\circ} 88^{\prime} 91^{\prime \prime} \mathrm{E}$ \\
\hline 4. & $2000-1600 \mathrm{BC}$ & Arkaim & $52^{\circ} 38^{\prime} 57^{\prime \prime} \mathrm{N} 59^{\circ} 34^{\prime} 17^{\prime \prime} \mathrm{E}$ \\
\hline 5. & $1700 \mathrm{BC}$ & Mycenean Grave Circle B & $37^{\circ} 43^{\prime} 51^{\prime \prime} \mathrm{N} 22^{\circ} 45^{\prime} 23^{\prime \prime} \mathrm{E}$ \\
\hline 6. & $64 \mathrm{BC}$ & Varro's Aviary at Casinum (Tholos) & $41^{\circ} 29^{\prime} 24^{\prime \prime} \mathrm{N} 13^{\circ} 48^{\prime} 50^{\prime \prime} \mathrm{E}$ \\
\hline 7. & $31-28 \mathrm{BC}$ & Mausoleum of Augustus in Rome & $41^{\circ} 54^{\prime} 22^{\prime \prime} \mathrm{N} 12^{\circ} 28^{\prime} 35^{\prime \prime} \mathrm{E}$ \\
\hline 8. & $118-138$ & Teatro marittimo-Villa Hadriana & $41^{\circ} 94^{\prime} 24^{\prime \prime} \mathrm{N} 12^{\circ} 77^{\prime} 58^{\prime \prime} \mathrm{E}$ \\
\hline 9. & $766-7$ & Round city of Baghdad & $33^{\circ} 34^{\prime} 88^{\prime \prime} \mathrm{N} 44^{\circ} 33^{\prime} 53^{\prime \prime} \mathrm{E}$ \\
\hline 10. & 836 & Large serdab Dar al-Khalifa, Samarra & $34^{\circ} 23^{\prime} 05^{\prime \prime} \mathrm{N} 43^{\circ} 87^{\prime} 40^{\prime \prime} \mathrm{E}$ \\
\hline 11. & $859-892$ & Tell al-Banat & $34^{\circ} 44^{\prime} 76^{\prime \prime} \mathrm{N} 43^{\circ} 80^{\prime} 48^{\prime \prime} \mathrm{E}$ \\
\hline 12. & 980-989 & The Early Slavic pagan shrine in Peryn & $58^{\circ} 47^{\prime} 28^{\prime \prime} \mathrm{N} 31^{\circ} 27^{\prime} 36^{\prime \prime} \mathrm{E}$ \\
\hline 13. & $1183-1186$ & Studenica Monastery, Serbia & $43^{\circ} 29^{\prime} 11^{\prime \prime} \mathrm{N} 20^{\circ} 32^{\prime} 13^{\prime \prime} \mathrm{E}$ \\
\hline 14. & 1240 & Harman Fortified Church & $45^{\circ} 42^{\prime} 56^{\prime \prime} \mathrm{N} 25^{\circ} 41^{\prime} 06^{\prime \prime} \mathrm{E}$ \\
\hline 15. & 1499 & Hypnerotomachia PoliphiliIsland garden of Cythera & imagination \\
\hline 16. & 1545 & Orto Botanico di Padova & $45^{\circ} 39^{\prime} 12^{\prime \prime} \mathrm{N} 11^{\circ} 88^{\prime} 08^{\prime \prime} \mathrm{E}$ \\
\hline 17. & 1593 & Palmanova & $45^{\circ} 90^{\prime} 56^{\prime \prime} \mathrm{N} 13^{\circ} 31^{\prime} 00^{\prime \prime} \mathrm{E}$ \\
\hline 18. & $1630-1665$ & Parc d'Enghien & $50^{\circ} 68^{\prime} 76^{\prime \prime} \mathrm{N} 04^{\circ} 04^{\prime} 73^{\prime \prime} \mathrm{E}$ \\
\hline 19. & $1731-1746$ & The Palace Garden Karlsruhe & $49^{\circ} 01^{\prime} 38^{\prime \prime} \mathrm{N} 08^{\circ} 40^{\prime} 44^{\prime \prime} \mathrm{E}$ \\
\hline 20. & $1737-1747$ & Clemenswerth Palace, Sögel & $52^{\circ} 83^{\prime} 83^{\prime \prime} \mathrm{N} 07^{\circ} 53^{\prime} 71^{\prime \prime} \mathrm{E}$ \\
\hline 21. & $1753-1770$ & Schwetzingen Palace, Schwetzingen & $49^{\circ} 38^{\prime} 37^{\prime \prime} \mathrm{N} 08^{\circ} 56^{\prime} 80^{\prime \prime} \mathrm{E}$ \\
\hline 22. & 1770 & ErylouHua'an tuloi, Hua'an, Zhangzhou, Fujian, China & $25^{\circ} 01^{\prime} 25^{\prime \prime} \mathrm{N} 117^{\circ} 41^{\prime} 12^{\prime \prime} \mathrm{E}$ \\
\hline 23. & 1771 & Șarlota (Charlottenburg) & $45^{\circ} 97^{\prime} 86^{\prime \prime} \mathrm{N} 21^{\circ} 51^{\prime} 91^{\prime \prime} \mathrm{E}$ \\
\hline 24. & 1836 & Circleville, Ohio & $39^{\circ} 35^{\prime} 58^{\prime \prime} \mathrm{N} 82^{\circ} 56^{\prime} 40^{\prime \prime} \mathrm{W}$ \\
\hline 25. & 1930s & Queen Mary's Gardens Regent's Park, London & $51^{\circ} 52^{\prime} 77^{\prime \prime} \mathrm{N} 00^{\circ} 15^{\prime} 36^{\prime \prime} \mathrm{W}$ \\
\hline 26. & 1964 & Brøndby Garden City, Copenhagen & $55^{\circ} 38^{\prime} 13^{\prime \prime} \mathrm{N} 12^{\circ} 23^{\prime} 58^{\prime \prime} \mathrm{E}$ \\
\hline 27. & 1970 & Kikar Hamedina, Tel Aviv & $32^{\circ} 05^{\prime} 12^{\prime \prime} \mathrm{N} 34^{\circ} 47^{\prime} 23^{\prime \prime} \mathrm{E}$ \\
\hline 28. & 1972 & Bublik Circular House, Moscow & $55^{\circ} 42^{\prime} 26^{\prime \prime} \mathrm{N} 37^{\circ} 28^{\prime} 16^{\prime \prime} \mathrm{E}$ \\
\hline 29. & contemporary & Maheshwari Udyan, Mumbai & $19^{\circ} 02^{\prime} 67^{\prime \prime} \mathrm{N} 72^{\circ} 85^{\prime} 54^{\prime \prime} \mathrm{E}$ \\
\hline 30. & contemporary & Gartenverein zur Sonnenblume Kamp-Lintfort & $51^{\circ} 49^{\prime} 15^{\prime \prime} \mathrm{N} 06^{\circ} 56^{\prime} 54^{\prime \prime} \mathrm{E}$ \\
\hline 31. & contemporary & Upper Midwest Circle City & $43^{\circ} 04^{\prime} 00^{\prime \prime} \mathrm{N} 89^{\circ} 24^{\prime} 00^{\prime \prime} \mathrm{W}$ \\
\hline 32. & 2013 & Gasholder Park, London & $51^{\circ} 32^{\prime} 13^{\prime \prime} \mathrm{N} 0^{\circ} 07^{\prime} 41^{\prime \prime} \mathrm{W}$ \\
\hline 33. & 2015 & Navy Yard Central Green, Philadelphia & $39^{\circ} 89^{\prime} 38^{\prime \prime} \mathrm{N} 75^{\circ} 17^{\prime} 12^{\prime \prime} \mathrm{W}$ \\
\hline 34. & 2017 & Apple Campus 2, Cupertino & $37^{\circ} 33^{\prime} 50^{\prime \prime} \mathrm{N} 122^{\circ} 00^{\prime} 90^{\prime \prime} \mathrm{W}$ \\
\hline 35. & Pre-Columbian era & Bird enclosure earthwork at Kickapoo river, Wisconsin & $43^{\circ} 28^{\prime} 32^{\prime \prime} \mathrm{N} 90^{\circ} 41^{\prime} 38^{\prime \prime} \mathrm{W}$ \\
\hline 36. & Pre-Columbian era & Portsmouth Earthworks Group C, Kentucky & $38^{\circ} 44^{\prime} 09^{\prime \prime} \mathrm{N} 82^{\circ} 54^{\prime} 12^{\prime \prime} \mathrm{W}$ \\
\hline
\end{tabular}

\subsubsection{Antiquity}

The moment of transition from circle to polygonal shaped structures can be sought in the period when large settlements are formed-cities, and later states-through urban revolution [20]. The first cities were created in Mesopotamia in 3000 BC [21]. Edmund Burke notes that Man's dwellings are the most appropriate and solid when they are in a polygonal 
form, and that the pieces fit together easily [22]. However, there are heterogeneous circular structures that can be found throughout the old ages.

In the southern Trans-Urals were a number of middle Bronze Age fortified settlements of the Sintashta-Arkaim culture. The sites are characterized by settlements with closed double fortification walls and two circles of houses, separated by a street and a central square free of buildings. The most significant remains of that kind include Sintashta, Arkaim, Bersuat, Isiney, Sarym-Sakly and Zhurumbay [23,24].

In ancient Greece, elements of a circle are encountered in the amphitheater. The orchestra's area measured about $25 \mathrm{~m}$ and was in a circular shape at the foot of a slope along which rows of seats were arranged in a semicircular manner [25].

This concept of the amphitheater has been altered, and traces of possible alterations can be found in the writings of Pliny the Elder. He describes the creation of C. Scribonius Curio from 53 or $52 \mathrm{BC}$. It consisted of two semicircular theatres that could rotate and form an arena. This amphitheater is the harbinger of later circuses, stadiums, arenas and hippodromes. It indicates the possible evolution of the Greek semicircular into the Roman circular amphitheater (Figure 5).

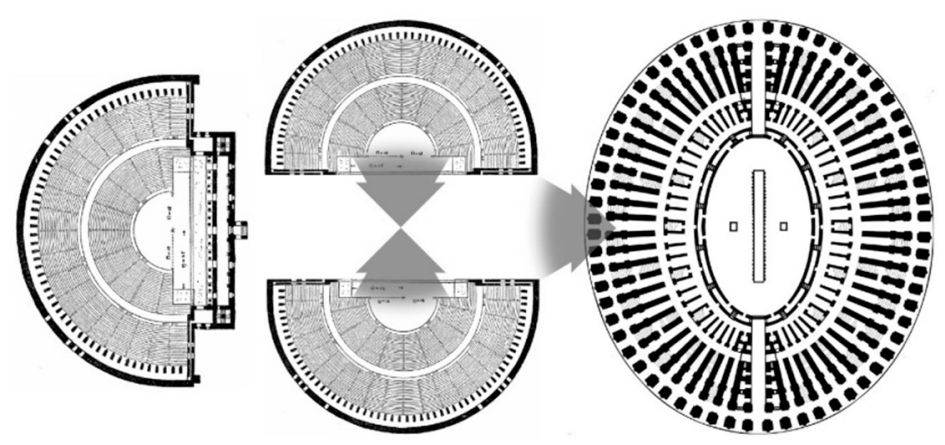

Figure 5. Hypothetical scheme of evolution of Old Greek amphitheater to Roman one and some exemplars of ancient and contemporary amphitheaters.

Varro's Aviary, built at Casinum by Marcus Terentius Varro, consists of two main parts, one square and one round. The round domed structure, the tholos, was surrounded with a colonnade. Its outer pillars were made of stone, and the inner ones of living trees; these were used for cages. A narrow stone path divides the colonnade from the round canal inside that held a variety of birds [26].

The only exception is the Mausoleum of Augustus in Rome within which the planting of trees on the peripheral part, that is, on the lower annular part of the roof, was carried out. The description in Strabo Geography is very illustrative: "The most noteworthy is what is called the Mausoleum, a great mound near the river on a lofty foundation of white marble, thickly covered with evergreen trees to the very summit. Now on top is a bronze image of Augustus Caesar; beneath the mound are the tombs of himself and his kinsmen and intimates; the wall which round his crematorium is surrounded by a circular iron fence and the space within the wall is planted with black poplars" [27-29].

At Hadrian's villa in Tivoli is the Teatro Marittimo circular island, accessed by two drawbridges. A circular wall of tuff and brick outside the canal forms, along with 40 ionic white marble columns, an internal portico, concentric with a wide and deep marble tiled channel [30].

\subsubsection{Middle Ages}

Since the collapse of the Roman Empire in 476, Roman style buildings and gardens in Europe became more decentralized, deurbanized and agrarian. Circular structures are rare because of the cramped space in cities, monasteries and castles. If they exist, they are simple and with plenty of BUA. 
The Early Slavic pagan shrine in Peryn, south of Novgorod, an example of a primitive structure with a circular floor plan, belongs to the Early Medieval period. The sanctuary was surrounded by a ditch $1 \mathrm{~m}$ deep. The trench had 8 semicircular flower petals with a radius of $7 \mathrm{~m}$. The petals were used for ritual fires (eternal flames), which is inferred from the findings of coal. In the center of the shrine was a statue of the god Perun [31].

The medieval Serbian Orthodox monastery of Studenica is surrounded by a circular stone wall with a temple in the center. The main entrance to the monastery is placed on the main east-west axis. The elliptical shape of the base occurs due to the slope along the north-south axis, making it a smaller axis of an ellipse, and proof of the incredibly accurate measurement of distance in the field [32].

In the absence of any archaeological remains of the Byzantine gardens, we cite the work of Theodore Hirtaken, a 14th century literatus, describing the garden of St Anna, the mother of the Virgin Mary, as a typical Byzantine garden, displaying his plan in a circle [33]. Hirtaken's description evokes a ring-shaped stone wall and several concentric circles of trees-choruses. The outer chorus was composed of cypresses, and several inner choruses of all kinds of fruit trees. In the center of the garden was a fountain with a pine cone in the middle.

Cvetković Tomašević (1973) describes a mosaic on the floor of the narthex of the Great Basilicain Heraclea Lyncestis (North Macedonia) from the early Byzantine period [34]. If the arrangement shown is seen as a radial cross-section of a circular garden, it does not fully fit with the earlier descriptions by which the "protective" trees are on the outer rings and edible fruits in the middle. What is common is the central decorative element, which here is a vessel with vines instead of a fountain, but surrounded by cypress trees. The next concentric circle, or chorus, consists of olive trees. The next chorus is apples and pears, and the periphery consists of cherries and figs, rather than cypresses as in the garden of St Anna. An outer circle then represents a "second periphery", consisting of cedar and pomegranate (Figure 6).

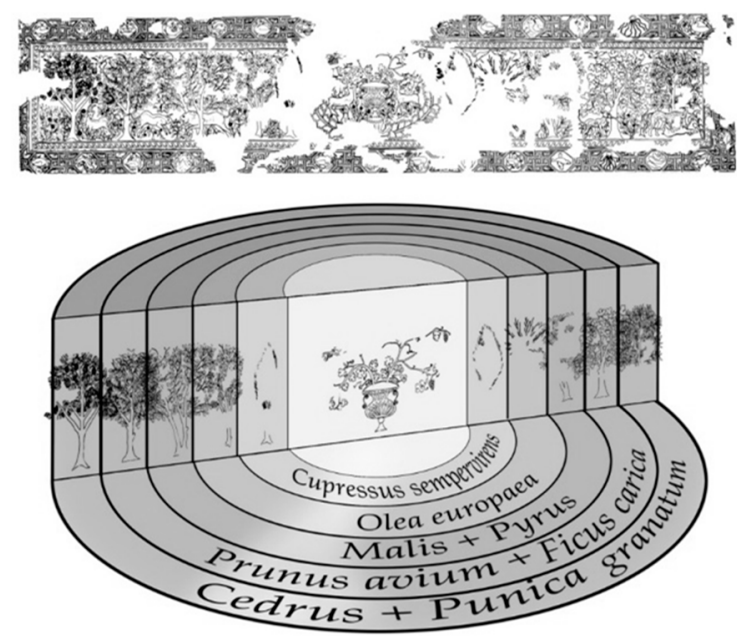

Figure 6. Possible appearance of 3D Byzantine Garden reconstructed from mosaic in Heraclea Lyncestis.

\subsubsection{Islamic Style}

One of these circular projects was the Round City of Baghdad, similar to the much older city of Mara. The Circular City had four main gates, at equal distances from each other. The walls were made of adobe brick with a girder, while the domes and vaults were of baked brick [35]. The space between the outer and inner wall was occupied by houses and shops and divided into quadrants [36,37]. The space from the inner wall to the central buildings (palace, a mosque, residences for princes and offices) was a garden. Unlike Mari, 
the zones of Baghdad are reversed, with green spaces separating the peripheral residential area from the palace and mosque in the center.

In Samarra, the city that was the capital of the Abbasid Caliphate from 836 to 892, Dar al-Khilafa, a palace complex, was erected. In the complex is an easily recognizable circular structure named the Large Serdab (or Birka Da'iriyya), a sunken basin without an OS and with subterranean chambers around it [38].

Two kilometers southeast of al- Dur there is a circular artificial mound, Tell al-Banat, standing $18.2 \mathrm{~m}$ above the surrounding plain. The canal of al-Mutawakkil circles the base of the mound. On the mound, a pavilion may have existed. Susa (1948-9) mentions the remains of bricks [39].

\subsubsection{Renaissance}

Leon Battista Alberti, one of the greatest contributors to Renaissance ideas on architecture, in his classic treatise De re aedificatoria (On the Art of Building), begins with the ideal form of the circle, which is expressed in numerous examples of nature. In Alberti's work De Architectura, he desires to see circles and semicircles, considered beautiful in villas, in the garden as well, and these he would have made with laurel, citron, and yew, with interwoven branches [40].

In Cortile del Belvedere, at the Vatican Palace in Rome finished by Pirro Ligorio in 1562-65, designer Bramante continued with Alberti's concept of the application of circles or, more precisely, semicircles as building and garden details. The exedra on the facade of the Villa Belvedere and the nicchione on the top are semicircular elements of the upper end of the court. On the opposite, southern, end was an amphitheater attached to the main palace and corresponding to the exedra and nicchione. With seats for spectators in the amphitheater, this lower part of the courtyard could be used for entertainment and performances (Figure 7).

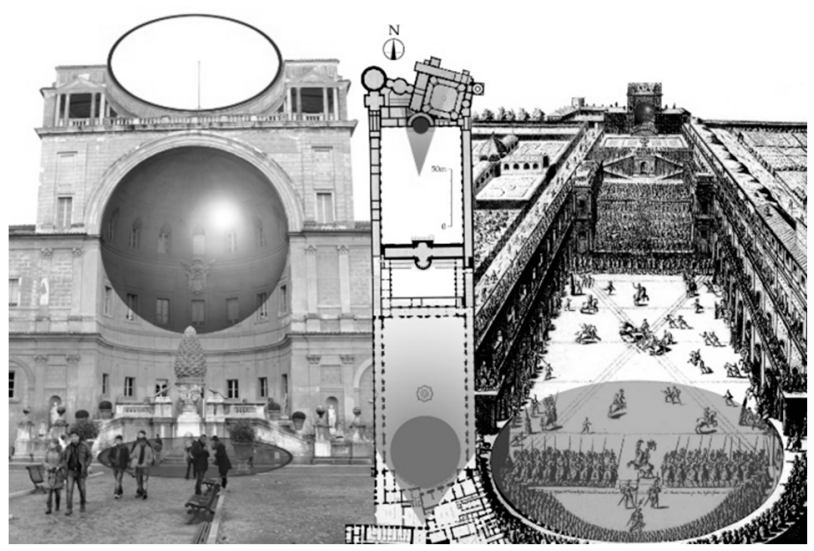

Figure 7. Spherical and circular elements in Cortile del Belvedere (Belvedere Courtyard) at the Vatican Palace.

\subsubsection{Baroque}

Baroque style and compositional features are expressed through absolute symmetry, geometry, monumentality and central perspective. The geometry is dominated by a circle, a trapezoid or an ellipse, and the internal and external organization of the space interconnect one another [41].

\subsubsection{Contemporary Era and Circle City Idea}

Gartenverein Zur Sonnenblume, Kamp-Lintfortis' garden society is composed of about 70 plots of land made available for individual, non-commercial gardening or for growing food in a community garden. The society advocates an ecological approach and propagates a natural kind of life through gardening and beekeeping. The space is divided into radially concentric parcels [42]. 
The designers of the Navy Yard Central Green Park are James Corner Field Operations. The circular segments, from the designers' vision, are original designs of nature. Central Green provides spaces for relaxation and escape and for exercise and recreation for 11,000 employees of companies of the 1200 acre Navy Yard community. The circular park division appears to have separate outdoor rooms that create a multi-purpose space. The uses include a fitness station, an amphitheater/"sun lawn", a hammock grove, bocce courts, ping-pong tables, a communal table, and a "bio-basin" for storm water. The vision is similar to Garden Cities [43]; to build a sense of a cohesive mixed-used village integrating jobs and residents. Here exists a transition from the dull cubicle working lifestyle to an urban oasis within a commercial zone that boasts a great quality of life.

Apple Campus 2, Cupertino was erected from an idea of Steve Jobs, the co-founder of Apple Inc., to consolidate his employees. He wanted to create a new campus in the suburb of Cupertino, California, where the border between nature and the circular building would be blurred. The total area of the complex is $71 \mathrm{ha}$, but for our investigations we have taken into account only the area of the circular building and the space inside it. There are 9000 trees on the Apple Park campus, with 309 varieties of indigenous species. Due to its circular shape and size, the Apple Campus has been nicknamed "the spaceship" [44].

Circle Cities is an idea by Philip H. Lewis Jr, the founder and director of the Madison Environmental Awareness Center (EAC), based on North America Night-time Satellite Imagery and Urban Partial Illumination (BUA) versus Dark Conserved Nature (OS). To connect the illuminated parts, Lewis used ring megalopolises that surround natural landscapes. One of the circular cities is the Upper Midwest Circle City $\left(43^{\circ} 04^{\prime} 00^{\prime \prime} \mathrm{N}, 89^{\circ} 24^{\prime} 00^{\prime \prime} \mathrm{W}\right)$, which is $500 \mathrm{~km}$ in diameter and covers an area of $187,753 \mathrm{~km}^{2}$. The center of the circle is the Kickapoo Valley, WI within which the biodiversity is at its greatest, with the cities of Chicago, Milwaukee and Minneapolis located peripherally (Lewis, 1996). This trend should be maintained and urbanization within the "ring" of cities should not allowed. It connects US cities in a similar way with a methodology for guiding development in a manner that preserves and enhances natural diversity and quality of life for present and future generations (Figure 8) [45].

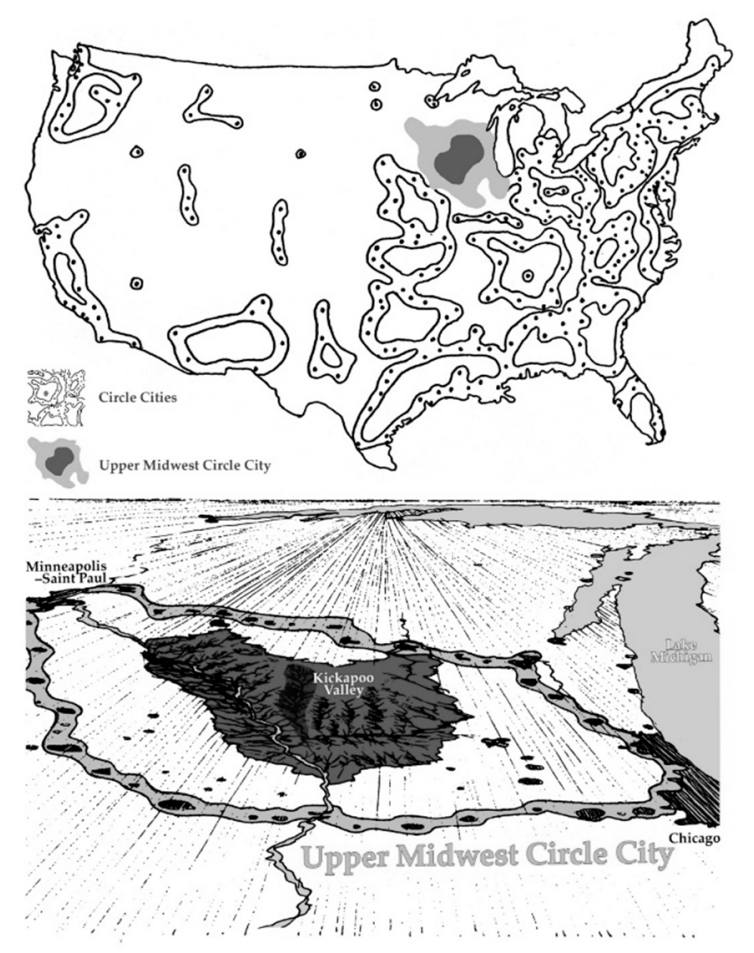

Figure 8. Circle Cities and Upper Midwest Circle City as idea of Philip H. Lewis. 


\section{Results and Discussion}

The results of morphospace analysis are presented in Table 4. Analyzing all three chosen variables, categories and types of circular objects, Jam area and \% of Jam and comparing these data with the historical timeline, we tried to explore if there is a ratio between OS and BUA and its historical interpretation. The morphospace analysis of this research indicates that there is no clear regularity in the relationship between the built-up area and the open space.

As we could see in Table 4, all circular objects are divided into two main categories: Objects with a Total Diameter Smaller than $300 \mathrm{~m}$ and Objects with a Total Diameter Larger than $300 \mathrm{~m}$. These categories are made so all selected circular objects could be studied as potential examples for circular building designs for different ratios of urban environments (smaller cities and areas, and larger urban spaces-circular cities studies) [46].

All circular objects are also split according to the type of open space (seven types): Parks-Gardens, Settlements, Earthworks, Sacral Objects, Circular Buildings, Allotments and Plazas. It is important to represent the types of selected circular objects as there is a difference between parks, settlements and, for example, large circular buildings, which will be explained further in the text.

Table 4. The representation of three variables of morphospace analysis of selected circular objects. Gray color represents value of $\%$ of Jam below $50 \%$, and green color-above $50 \%$. All images are from free Wikimedia Commons media repository.

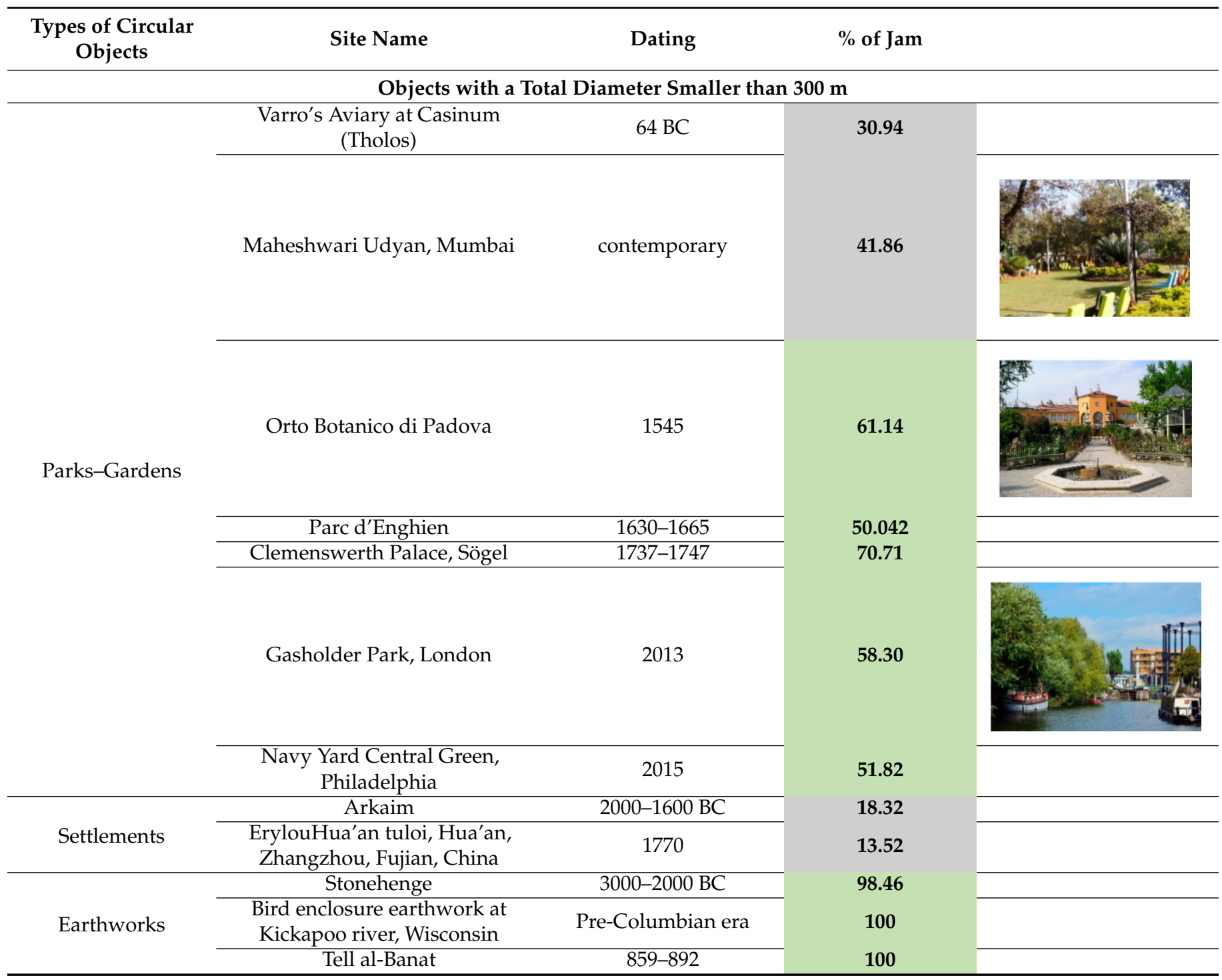


Table 4. Cont

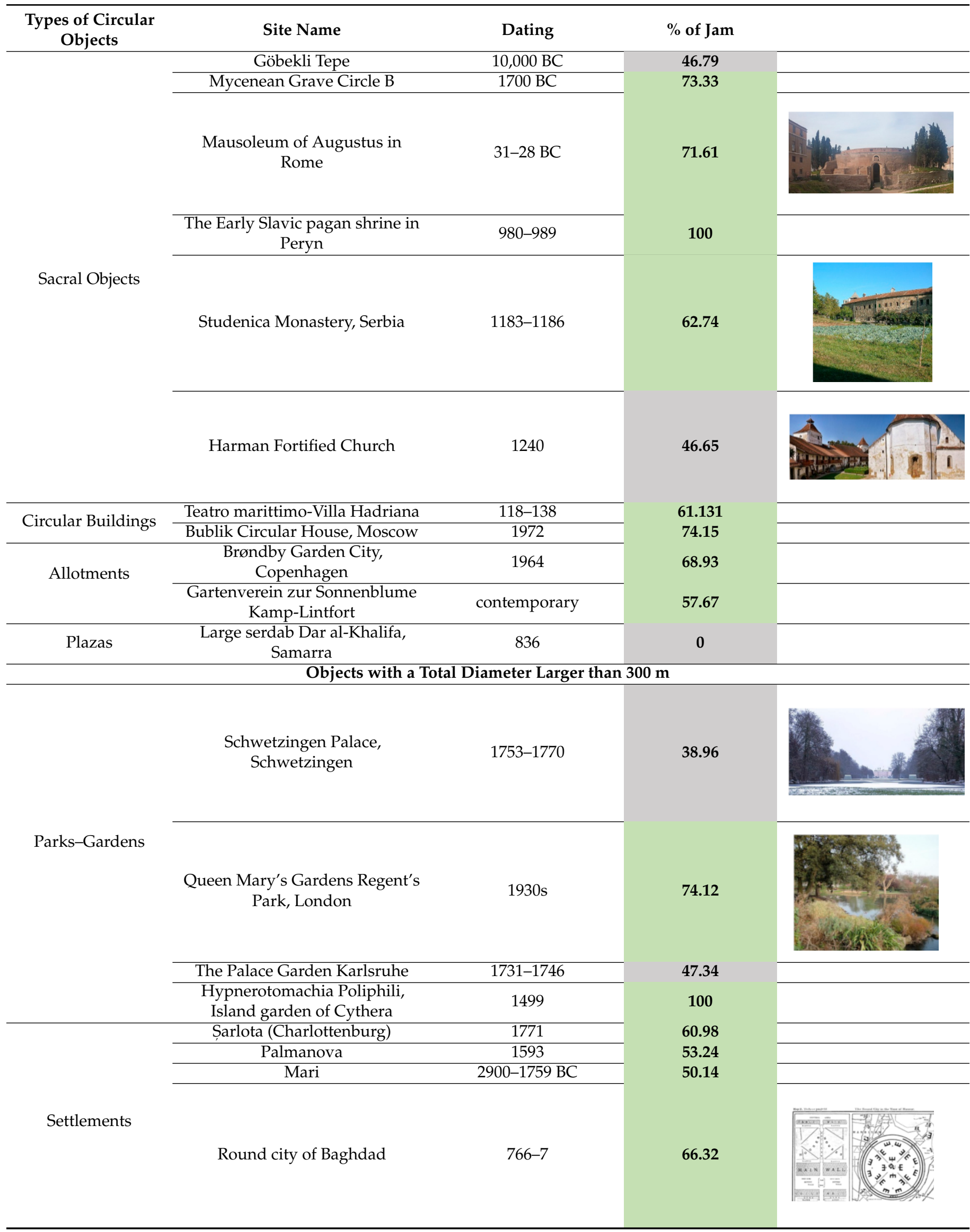


Table 4. Cont.

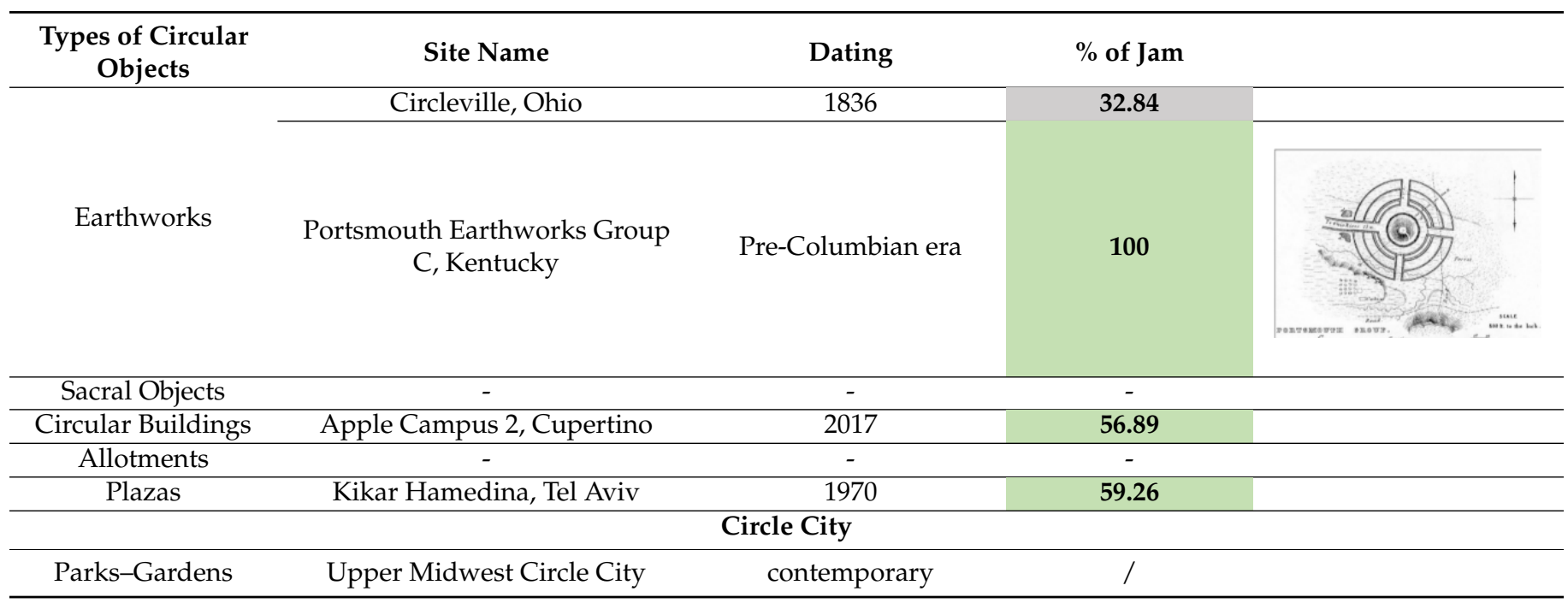

In the category of Parks and Gardens, the dimensions of these green spaces range from $223 \mathrm{~m}^{2}$ for Varro's Aviary at Casinum to 1,724,114 $\mathrm{m}^{2}$, the size of the imaginary island Garden of Cythera. The total area to OS ratio ranged from 31\% for Varro's Aviary to 100\% for the Garden of Cythera. In other gardens, the OS ranged from 39 to $74 \%$ (Table 1). The ratio of the total diameter to the inner diameter (morphospace) is smallest at Schwetzingen Palace with $62.42 \%$, followed by Varro's Aviary with $64.28 \%$ and Karlsruhe with $68 \%$; the inner diameters of Parc d'Enghien and Maheshwari Udyan represent $69 \%$ of the total diameter. For Navy Yard Central Green, Orto Botanico di Padova, and Gasholder Park, the ratio is 71 to $78 \%$. The highest percentages were recorded at Clemenswerth Palace (84\%), Queen Mary's Gardens (86\%) and the Garden of Cythera, where the entire area is OS, so the overall diameter and inner diameter are the same.

In the category of Settlements, the total area to OS ratio ranges from $50 \%$ at Mari to $66 \%$ at the Round City of Baghdad, while the OS at Palmanova was $54 \%$. The ratio of the total and inner diameters is 71\% for Mari, 72\% for Baghdad and 73\% for Palmanova. Smaller settlements are included in the same category. This work covers three such spaces built from 2000-1600 BC to 1771. The first is Arkaim, the second is Sarlota, and the third fortified settlement is the ErylouHua'an tuloi. Settlement areas are significantly smaller than fortified cities, being $16,917 \mathrm{~m}^{2}$ for Arkaim and $4231 \mathrm{~m}^{2}$ for ErylouHua'an tulou, while Șarlota is much larger $\left(136,639 \mathrm{~m}^{2}\right)$.

The OS is significantly smaller in the fortified settlements of Arkaim and ErylouHua'an tuloi, at $18 \%$ and $14 \%$, respectively, while in Șarlota the OS is $61 \%$. Morphospace analysis indicates that the inner diameter is less with a fortified settlement (37\% with ErylouHua'an tuloi and $42 \%$ with Arkaim) than with Șarlota (78\%).

In the category of Earthworks, as a rule, the earthwork's OS is the only surface confirmed by Tell al-Banat, the Bird enclosure earthwork and Portsmouth Earthworks. With Stonehenge's OS, the $98 \%$ ratio is smaller for the surface on which stone monoliths lie. Circleville had a $100 \%$ OS before the settlement was established, and with urbanization in the 19th century the OS fell to $33 \%$, and today it is certainly even smaller.

The morphospace analysis in this group indicates a specific diameter ratio, with total and inner diameter coinciding (100\%) with pure earthworks, and varying from $56 \%$ in Circleville to $99 \%$ in Stonehenge.

In the category of Sacral Objects, the OS is relatively the largest in Peryn (100\%), followed by the Mycenean Grave Circle B (73\%), the Mausoleum of Augustus (71\%), Studenica (63\%), and it is the lowest in Göbekli Tepe and Harman (47\%). 
The ratio of the total diameter to the metaphorical "jam diameter" is at Peryn (100\%) followed by the Mycenean Grave Circle B (85\%), the Mausoleum of Augustus (84\%), Studenica $(73 \%)$, and Göbekli Tepe $(70 \%)$, while the smallest OS is at Harman.

In the category of Circular Buildings, the OS is largest at Bublik with 74\%, followed by the Teatro Marittimo (61\%), with Apple Campus 2 having the smallest OS in the circular buildings category (57\%).

In the category of Allotments, it is logical that in this type most of the space is occupied by the OS, but the part leading to the OS is not significantly different from the OS in other groups. The OS of Gartenverein zur Sonnenblume is 58\% and it is $69 \%$ at Brøndby Garden City. The metaphorical "jam" diameters are $76 \%$ and $82 \%$, respectively.

In the category of Plazas, two plazas were taken into consideration-Large Serdab in Samarra and Kikar Hamedina in Tel Aviv. Kikar Hamedina is a contemporary (1970) object with an area of $86,484 \mathrm{~m}^{2}$ and an area to OS ratio of $59 \%$, with $77 \%$ of the diameters belonging to the "jam". Large Serdab is part of Dar al-Khalifa, erected in 836, in the time of Abbasid Samarra. Its area is $1962 \mathrm{~m}^{2}$, without an OS.

In Table 4, for the purpose to represent some potentially good examples which have more than $50 \%$ of Jam area-open space of circular objects, we selected two colors: green color (for the \% values higher than 50\%) and gray color (for the \% values lower than 50\%).

Evaluating this value in Table 4, from 35 selected circular objects for this research (Circle City excluded), 25 circular objects have more than $50 \%$ of open space in both categories. The 10 circular objects have lower than $50 \%$ of open space in both categories, and in this analysis Upper Midwest Circle City was excluded because it was an example of the Circle City idea-large space area. This could show the potential of using circular objects as a city form in future urban development, as more than $50 \%$ of selected circular objects had more than $50 \%$ of open space.

Evaluation by chosen categories has shown a ratio in the category Objects with a Total Diameter Smaller than $300 \mathrm{~m}$ of 16:7, and in the category Objects with a Total Diameter Larger than $300 \mathrm{~m}$ of 9:3. In first category, we could also see that more than $50 \%$ of selected circular objects had values above $50 \%$ of open space and that seven objects had below values. In the second category, the ratio 9:3 shows the same conclusion.

The analysis according to type of open spaces was best presented in graphical representations below.

This research represents a study of the relationship of open spaces (OSs) and built-up areas (BUAs) through history. One thing that could be concluded is that the larger \% of open space area could be achieved by using circular shapes-based on Figure 4, many selected circular objects have a ratio in favor of open spaces, and especially in "green" types of open spaces such as parks, gardens, earthworks and others. Other types of open spaces such as settlements, circular objects and plazas have around 50-65\% of Jam area.

In the context of sustainable design, the main value in this research was the \% of Jam. The reason for that is that this chosen value represents the $\%$ of open space in selected circular objects. The \% of Jam of all circular objects divided into two main categories is presented in Figures 9 and 10. In these graphical representations, we could see that two types of open spaces in both categories had high values of \% of Jam: Parks and Gardens and Earthworks. The lower values had types of spaces such as Settlements, Allotments and Plazas. In these graphical presentations, high values of \% of Jam had selected circular objects in the type Sacral Objects, which could be an interesting observation, and which could confirm the possibility of using circular building forms as new urban forms for sustainable urban development.

The high \% of open spaces in urban environments represents one of the sustainable strategies towards sustainable urban living, as the increase in this value has many positive outcomes to especially environmental aspects of urban environment [47-49]. There are many sustainable performances that different buildings in urban environments have to ensure, such as sustainable energy sources, water management, waste management and sustainable materials used, and for other aspects: health and safety, circular economy 
and other performances [50-53]. For the purpose of this research and in the aspect of exploring circular designs as possible sustainable urban forms which could possible ensure the increase in porous spaces in urban environments, the value \% of Jam explored in this research was important. The increase in porous spaces in cities has, for example, big significance for urban ventilation, air quality, soil quality and others. Additionally, social benefits are also important, in the form of recreational spaces, parks, urban gardening and many others. The increased \% of porous spaces in cities is one of the most important sustainable strategies in the climate change adaptation of cities [54-57].

Proof of using circular design through history could be clearer if these three objects, Bramante's tempietto, The Radcliff Camera and Sanaa's 21st Century Museum of Contemporary Art, currently not included in this research, are subjects of our future interest since they are obvious examples of using circular design in different periods and places.

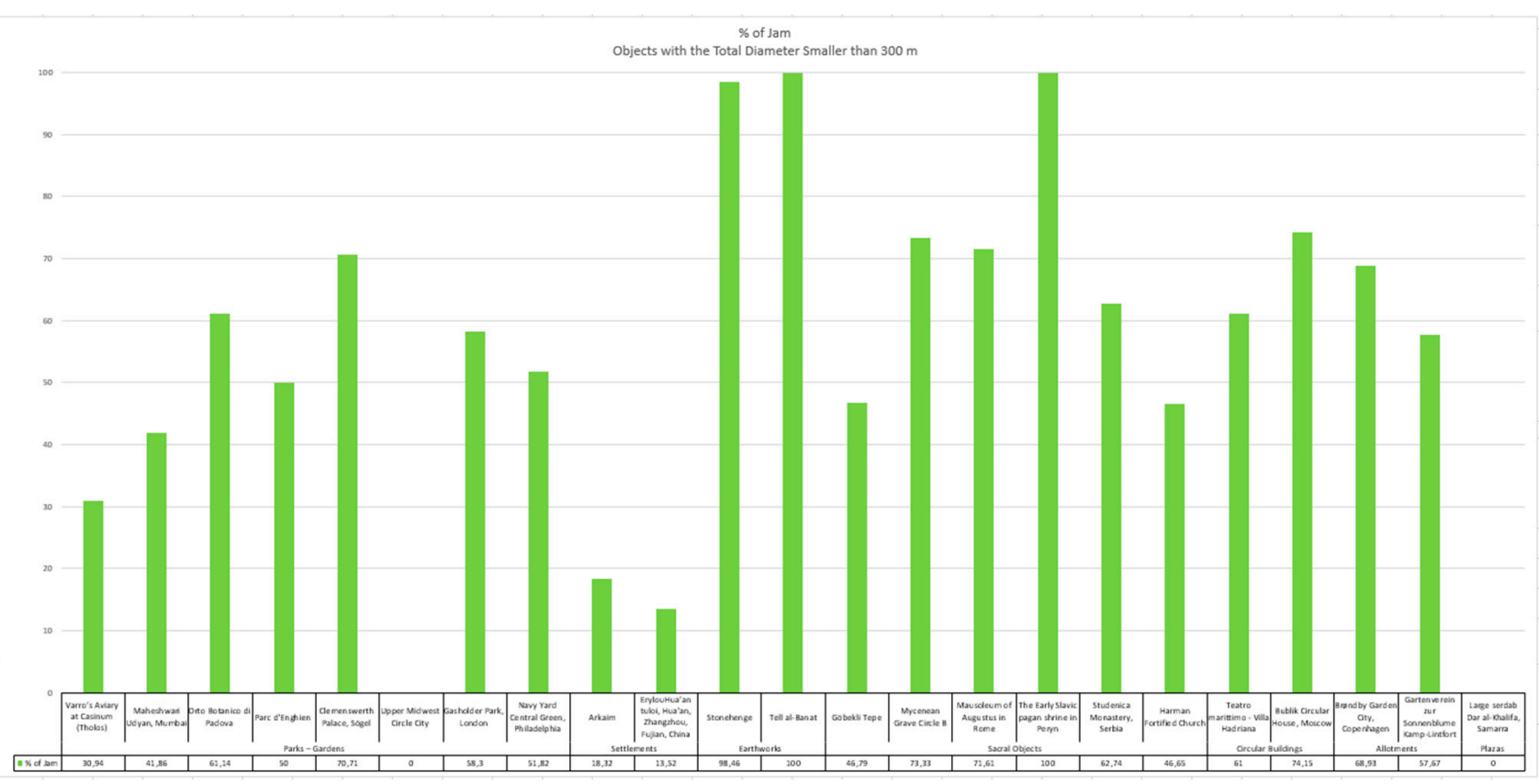

Figure 9. The graphical representation of selected circular objects with a total diameter smaller than $300 \mathrm{~m}$.

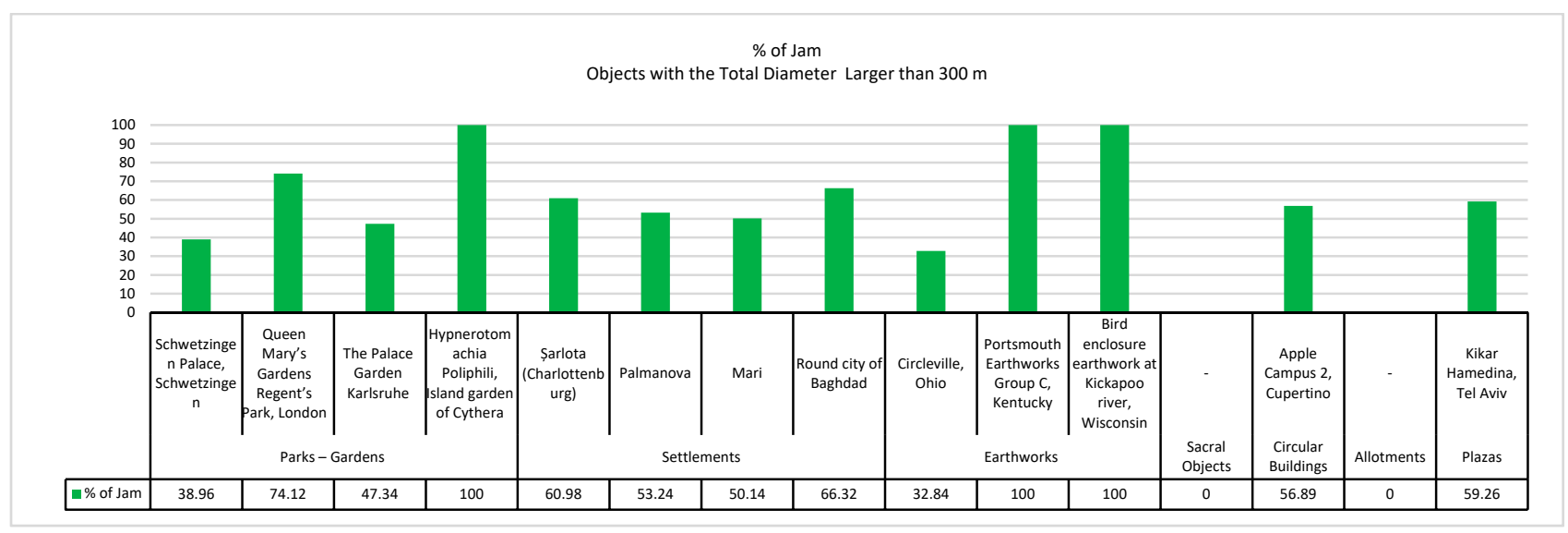

Figure 10. The graphical representation of selected circular objects with a total diameter larger than $300 \mathrm{~m}$.

The future circular design process could bring more stability, and the ratio between OS and BUA could be more balanced [58]. 
The new role of the architects as well as landscape architects could be the main actor for driving circular design by linking innovative production to circular economy principles [59].

Sustainability indicators such as environmental, socio-cultural, economic, and institutional indicators for urban development should include more parameters related to green open spaces, not only historical ones [60] but also circular design.

This study analyzes a number of historical circular objects with the aim of creating sustainable urban environments as a key ingredient for cities living sustainability.

\section{Conclusions}

The morphospace method makes it possible to strategically look at the range of possibilities for realizing circular space plans and the share of the built-up area (BUA) and open space (OS) within it. Of the 36 existing circular spaces analyzed, variants occur from those for which the entire space goes to BUA to those for which only an OS exists. This covers the full range of possible variants of the OS/BUA ratio, that is, the limit of analysis. From the perspective of certain categories of circular objects, no regularity can be observed in these relationships, with the exception of earthworks, where the proportion of OS is above $90 \%$. The largest variations in the relationship are in parks and gardens, where the OS ranges from 30-100\%. There are no regularities in the OS/BUA relationships over a long period of time from the Neolithic to the modern era, and it cannot be said that the percentage of OS increases or decreases. The same can be said about the size of circular objects.

As circular design and processes continue to have a main role in many sustainable strategies, the idea of circular cities as a future urban model will be more popular. An important part of every urban environment is open spaces in all their types, especially green spaces. The need for increasing the number of urban greenery and spaces is present in all cities around the world.

As there is a trend of increasing open spaces in city plans around the world and providing more spaces and ways of integrating urban greenery is one of the sustainable goals, this research could be used as a base for further research on circular forms and shapes. Many selected circular objects have a ratio in favor of open spaces through the history timeline, which is shown by this research, so the circular/doughnut shape could be a future reinvented pattern for urban development. Additionally, the observed occurrence of the disappearance of circular forms with urbanization, which is evident as a result of the space deficit and the difficulty in fitting circular forms together with one another or with other forms, cannot be fully accepted. The circular form will move to different levels, such as the mega spaces of Circle Cities. In addition, exploration beyond planet Earth and the conquering of outer space again gives room for circular shapes, and they are also inevitable as the only possibility for producing artificial gravity by their rotation and the influence.

Little is known about the benefits of circular designed spaces despite our understanding of the benefits of urban green spaces. The research results shown here highlighted the importance of circle design use with regard to citizens' well-being by environmental sustainability. A key component of sustainability is urban circular spaces providing optimal psychological and social benefits by raising citizens' quality of life.

Author Contributions: Conceptualization, M.N.G., B.S.J. and A.A.Č.; methodology, B.S.J.; software, M.N.G.; validation, B.S.J., A.A.Č. and M.N.G.; formal analysis, A.A.Č.; investigation, B.S.J.; resources, M.N.G.; data curation, A.A.Č.; writing-original draft preparation, B.S.J.; writing-review and editing, A.A.Č.; visualization, M.N.G.; supervision, B.S.J.; project administration, A.A.Č.; funding acquisition, M.N.G. All authors have read and agreed to the published version of the manuscript.

Funding: This research received no external funding.

Institutional Review Board Statement: Not applicable.

Data Availability Statement: Not applicable. 
Acknowledgments: The authors are supported by the Ministry of Education, Science and Technological Development of the Republic of Serbia, Project No. TP 36008 (451-02-68/2020/14/2000169).

Conflicts of Interest: The authors declare no conflict of interest.

\author{
Abbreviations \\ BUA Built-Up Area \\ OS Open Space
}

\title{
References
}

1. Shishegar, N. The Impact of Green Areas on Mitigating Urban Heat Island Effect: A Review. Int. J. Environ. Sustain. 2014, 9, 119-130. [CrossRef]

2. Myers, M. Decision Making in Allocating Metropolitan Open Space: State of the Art. Trans. Kans. Acad. Sci. 1975, $78,149$. [CrossRef]

3. Boeri, A.; Gaspari, J.; Gianfrate, V.; Longo, D.; Boulanger, S.O. Circular City: A Methodological Approach for Sustainable Districts and Communities; WIT Transactions on the Built Environment; WIT Press: Southhampton, UK, 2019; Volume 183, pp. 73-82.

4. Marin, J.; De Meulder, B. Interpreting Circularity. Circular City Representations Concealing Transition Drivers. Sustainability 2018, 10, 1310. [CrossRef]

5. Schwerdtfeger, H. Geometry of Complex Numbers: Circle Geometry, Moebius Transformation, Non-Euclidean Geometry; Dover Publications, Inc.: New York, NY, USA, 1979; pp. 5-10.

6. Coolidge, J.L. A Treatise on the Circle and the Sphere. Math. Gaz. 1916, 8, 338. [CrossRef]

7. Casey, J. A Treatise of the Analytical Geometry of the Point, Line, Circle, and Conic Sections ; University College Dublin: Dublin, Ireland, 1893.

8. Culbert, C.; Ebert, G. Circle geometry and three-dimensional subregular translation planes. Innov. Incid. Geom. Algebr. Topol. Comb. 2005, 1, 3-18. [CrossRef]

9. Raup, D.M. Computer as Aid in Describing Form in Gastropod Shells. Science 1962, 138, 150-152. [CrossRef]

10. McGhee, G.R., Jr. The Geometry of Evolution-Adaptive Landscapes and Theoretical Morphospaces; Cambridge University Press: Cambridge, UK, 2006.

11. Steadman, P. Architectural Doughnuts: Circular-Plan Buildings, with and without Courtyards. Nexus Netw. J. 2015, 17, 759-783. [CrossRef]

12. Mitteroecker, P.; Huttegger, S.M. The concept of morphospaces in evolutionary and developmental biology: Mathematics and metaphors. Biol. Theory 2009, 4, 54-67. [CrossRef]

13. Martín, L.; March, L. Urban Space and Structures; Taylor \& Francis, Ltd.: Oxfordshire, UK, 1972.

14. Hilditch, M.H. Kepos: Garden Spaces in Ancient Greece: Imagination and Reality; Univerisity of Leicester: Leicester, UK, 2016.

15. Kural, B.D. Çaliş Three circular gardens in Venice and Constantinople/Istanbul in the context of early modern Mediterranean cross-cultural exchange. Stud. Hist. Gard. Des. Landsc. 2018, 38, 158-179. [CrossRef]

16. Turner, T. Garden History: Philosophy and Design 2000 BC-2000 AD; Routledge: New York, NY, USA, 2005.

17. Squier, E.G.; Davis., E.H. Ancient Monuments of the Mississippi Valley; Smithsonian Institution, GPO: Washington, DC, USA, 1848.

18. Van Cleaf, A.R. (Ed.) History of Pickaway County, Ohio, and Representative Citizens; Biographical Publishing Company: Chicago, IL, USA, 1906.

19. Xu, P. Feng-Shui Models Structured Traditional Beijing Courtyard Houses. J. Archit. Plan. Res. 1998, 15, 4.

20. Childe, V.G. Man Makes Himself; Watts \& Co. Library of Science and Culture: London, UK, 1936.

21. Spielvogel, J. Western Civilization: Volume A: To 1500; Cengage Learning: Boston, MA, USA, 2014; ISBN 978-1-285-98299-1.

22. Burke, E. A Philosophical Inquiry into the Origin of Our Ideas of the Sublime and Beautiful: With an Introductory Discourse Concerning Taste, and Several other Additions; Thomas M'lean, Haymarket: London, UK, 1823.

23. Bersenev, A.; Epimakhov, A.; Zdanovich, A. The Sintashta Bow of the Bronze Age of the South Trans-Urals, Russia from Bronze Age Warfare: Manufacture and Use of Weaponry; BAR International Series; Marianne, M., Uckelmann, M., Matthews, S., Eds.; Archaeopress: Oxford, UK, 2011; p. 2255.

24. Koryakova, L.; Kohl, P.L. Complex Societies of Central Eurasia from the 3d to the 1st Millennia b.c.: Regional Specifics in the Light of Global Models Source: Current Anthropology; The University of Chicago Press Journals: Chicago, IL, USA, 2000; Volume 41, pp. 638-642.

25. Liddell, H.G.; Scott, R. A Greek-English Lexicon; 9th Edition with Revised Supplement; Oxford University Press: Oxford, UK, 1996.

26. Van Buren, A.W.; Kennedy, R.M. Varro's Aviary at Casinum. J. Rom. Stud. 1919, 9, 63. [CrossRef]

27. Roller, D.W. The Geography of Strabo: An English Translation, with Introduction and Notes; Cambridge University Press: Cambridge, UK, 2017; ISBN 139781107038257.

28. Reeder, J.C. Typology and Ideology in the Mausoleum of Augustus: Tumulus and Tholos, Classical Antiquity; University of California Press: Oakland, CA, USA, 1992; Volume 11, pp. 265-307. 
29. Cordingley, R.A.; Richmond, I.A. The Mausoleum of Augustus, Papers of the British School at Rome; Cambridge University Press: Cambridge, UK, 1927; Volume 10, pp. 23-35.

30. Monaco, M.; Ranieri, M.; Lorenzo, C. Among the circles: A geometrical analysis of the Teatro Marittimo in Villa Adriana. In Proceedings of the SEAC 2010 Conference; BAR International Series 2794; BAR Publishing: Oxford, UK, 2016; pp. $225-229$.

31. Sedov, V.V. Drevnerusskoe Yazicheskoe Svyatilisce v Perini. Kratkie Soobsceniya Instituta Istorii Materialnoy kulturi; Institute of Archaeology Russian Academy of Sciences: Moscow, Russia, 1953; pp. 92-103.

32. Popović, S. Krst u krugu/Zaštita Manastira i Manastirsko Utvrđenje, Arhitektura Manastira u Srednjovekovnoj Srbiji; Prosveta, Republički Zavod za Zaštitu Spomenika Kulture: Beograd, Srbija, 1994; pp. 127-141.

33. Dolezal, M.-L.; Mavroudi, M.T. Hyrtakenos' Description of the Garden of St. Anna and the Ekphrasis of Gardens; Dumbarton Oaks, Trustees for Harvard University: Washington, DC, USA, 2002.

34. Cvetković Tomašević, G. Herakleja Linkestis, Odbor za Herakleju; Grafički Zavod: Bitola, Macedonia, 1973.

35. Creswell, K.A.C. A Short Account of Early Muslim Architecture; James, A., Aldershot, W., Eds.; Scolar Press, King's College: London, UK, 1989.

36. Ettinghausen, R.; Grabar, O. The Art and Architecture of Islam 650-1250, New Haven and London; Yale University Press: London UK, 1987.

37. Lassner, J. The Caliph's Personal Domain the City Plan of Baghdad Re-Examined. In The Islamic City; Hourani, S., Ed.; Bruno Cassirer Ltd.: Oxford, UK, 1970.

38. Northedge of the Palace of the Caliph at Samarra (Dar Al-Khilafa/ or Jawsaq Al-Khaqani). Ars Orient. 1993, $23,143-170$.

39. Susa, A. Rayy Samarra' fì 'Ahd al-Khilafa al-'Abbasiyya; Cambridge University Press: London, UK, 1948; Volume 2.

40. Gothein, M.-L. History of Garden Art, Hacker Art Books; Hacker Art Books: New York, NY, USA, $1972 ;$ ISBN 100878170081.

41. Grbić, M.; Čučaković, A.; Jović, B.; Tripković, M. Garden cultural heritage spatial functionalities: The case of anamorphosis abscondita at Vaux-le-Vicomte. J. Cult. Herit. 2016, 18, 366-369. [CrossRef]

42. MacNair, E. The Garden City Handbook: How to Create and Protect Community Gardens in Greater Victoria; Polis Project on Ecological Governance; University of Victoria: Victoria, BC, Canada, 2002.

43. Hall, P. Cities of Tomorrow, 3rd ed.; Blackwell: Malden, MA, USA, 2002.

44. Puddu, S.; Zuddas, F. Campus: Order and Exemption. Apple Campus 2, or the Mothership Has Landed in Cupertino; TRANS: Zurich, Switzerland, 2012.

45. Lewis, P.H. Tomorrow by Design: Regional Design Process for Sustainability; John Wiley and Sons: New York, NY, USA, 1996; ISBN 100471109355.

46. Deru, M.; Torcelini, P. Improving Sustainability of Buildings through a Performance-Based Design Approach. In Proceedings of the World Renewable Energy Congress VIII and Expo Denver, Denver, CO, USA, 29 August-3 September 2004.

47. Yuan, C.; Ng, E. Building porosity for better urban ventilation in high-density cities-A computational parametric study. Build. Environ. 2012, 50, 176-189. [CrossRef]

48. Gallagher, J.; Baldauf, R.; Fuller, C.H.; Kumar, P.; Gill, L.W.; McNabola, A. Passive methods for improving air quality in the built environment: A review of porous and solid barriers. Atmos. Environ. 2015, 120, 61-70. [CrossRef]

49. Robinson, J. Squaring the circle? Some thoughts on the idea of sustainable development. Ecol. Econ. 2004, 48, 369-384. [CrossRef]

50. Day, S.D.; Dickinson, S.B. (Eds.) Managing Storm Water for Urban Sustainability Using Trees and Structural Soils; Virginia Polytechnic Institute, and State University: Blacksburg, VA, USA, 2008. Available online: https://www.waynenyswcd.org/wp-content/ uploads/TreesAndStructuralSoilsManual.pdf (accessed on 22 March 2019).

51. Donofrio, J.; Kuhn, Y.; McWalter, K.; Winsor, M. Research Article: Water-Sensitive Urban Design: An Emerging Model in Sustainable Design and Comprehensive Water-Cycle Management. Environ. Pract. 2009, 11, 179-189. [CrossRef]

52. Jenks, M.; Dempsey, N. (Eds.) Future Forms and Design for Sustainable Cities; Routledge: Oxfordshire, UK, 2005; ISBN 9780750663090.

53. Ali, M.; Armstrong, P. Overview of Sustainable Design Factors in High Rise Buildings. 2008. Available online: https://global. ctbuh.org/resources/papers/download/1308-overview-of-sustainable-design-factors-in-high-rise-buildings.pdf (accessed on 15 June 2019).

54. Stijn, A.; Gruis, V. Towards a circular built environment: An integral design tool for circular building components. Smart Sustain. Built Environ. 2020, 9. [CrossRef]

55. Foster, G.; Saleh, R. The Adaptive Reuse of Cultural Heritage in European Circular City Plans: A Systematic Review. Sustainability 2021, 13, 2889. [CrossRef]

56. Cerreta, M.; Di Girasole, E.G.; Poli, G.; Regalbuto, S. Operationalizing the Circular City Model for Naples' City-Port: A Hybrid Development Strategy. Sustainability 2020, 12, 2927. [CrossRef]

57. Fan, Y.; Li, Y.; Yin, S. Non-uniform ground-level wind patterns in a heat dome over a uniformly heated non-circular city. Int. J. Heat Mass Transf. 2018, 124, 233-246. [CrossRef]

58. Jensen, K.G.; Sommer, J. Building a Circular Future; GXN: Copenhagen, Denmark, 2016.

59. Kanters, J. Circular Building Design: An Analysis of Barriers and Drivers for a Circular Building Sector. Buildings 2020, 10, 77. [CrossRef]

60. Rostami, R.; Lamit, H.; Khoshnava, S.M.; Rostami, R.; Rosley, M.S.F. Sustainable Cities and the Contribution of Historical Urban Green Spaces: A Case Study of Historical Persian Gardens. Sustainability 2015, 7, 13290-13316. [CrossRef] 